\title{
Mixing and entrainment in hydraulically driven stratified sill flows
}

\author{
By MORTEN HOLTEGAARD NIELSEN†, LARRY PRATT \\ AND KARL HELFRICH
}

Woods Hole Oceanographic Institution, MS 21, 360 Woods Hole Road, Woods Hole, MA 02543, USA

(Received 6 March 2003 and in revised form 21 May 2004)

The investigation involves the hydraulic behaviour of a dense layer of fluid flowing over an obstacle and subject to entrainment of mass and momentum from a dynamically inactive (but possibly moving) overlying fluid. An approach based on the use of reduced gravity, shallow-water theory with a cross-interface entrainment velocity is compared with numerical simulations based on a model with continuously varying stratification and velocity. The locations of critical flow (hydraulic control) in the continuous model are estimated by observing the direction of propagation of small-amplitude long-wave disturbances introduced into the flow field. Although some of the trends predicted by the shallow-water model are observed in the continuous model, the agreement between the interface profiles and the position of critical flow is quantitatively poor. A reformulation of the equations governing the continuous flow suggests that the reduced gravity model systematically underestimates inertia and overestimates buoyancy. These differences are quantified by shape coefficients that measure the vertical non-uniformities of the density and horizontal velocity that arise, in part, by incomplete mixing of entrained mass and momentum over the lowerlayer depth. Under conditions of self-similarity (as in Wood's similarity solution) the shape coefficients are constant and the formulation determines a new criterion for and location of critical flow. This location generally lies upstream of the critical section predicted by the reduced-gravity model. Self-similarity is not observed in the numerically generated flow, but the observed critical section continues to lie upstream of the location predicted by the reduced gravity model. The factors influencing this result are explored.

\section{Introduction}

The concept of hydraulic control plays an enormous role in understanding flow through a constriction and the influence it has on the basin circulation at both ends. Hydraulic theory was originally developed for engineering purposes, but with Stommel \& Farmer's (1953) study of estuary flow and Long's (1954) towing experiments, this phenomenon gained the attention of oceanographers and meteorologists. An appealing thought in current geophysical research is that hydraulic control may exist on the boundaries between the deep basins and marginal seas of the world's oceans and so be applied in the study of global thermohaline circulation and climate variability. Thus, from measurements of stratification in the Norwegian Sea, Hansen,

$\dagger$ Present address: Institute for Hydrobiology and Fisheries Science, University of Hamburg, Olbersweg 24, 22767 Hamburg, Germany.

$\ddagger$ Author to whom correspondence should be addressed: lpratt@whoi.edu. 
Turrell \& Østerhus (2001) have made suggestions about long-term changes of the dense flow of Arctic water into the North Atlantic.

Assumptions often made in hydraulic theories include the neglect of friction and mixing and that the water column consists of a number of layers, each having uniform density and velocity. Important results have been obtained in this way, including the significance of the composite Froude number and the concept of multiple controls (e.g. Farmer \& Armi 1986). Some elementary effects of friction have been discussed by Pratt (1986) who showed that simple quadratic bottom drag forces the flow towards criticality and displaces the control section downstream of its usual location (such as a sill or narrows). In naturally occurring flows, mixing can also play an important role. Prominent examples are the exchange flow through the Strait of Gibraltar, in which an intermediate layer is formed by mixing between the inflow and outflow (Bray, Ochoa \& Kinder 1995), and the Denmark Strait overflow, which is diluted as it descends into the North Atlantic (e.g. Käse \& Oschlies 2000; Girton, Sanford \& Käse 2001).

These examples show how mixing works to alter vertical gradients and induce changes in the flow properties in the along-channel direction. In an attempt to capture the essence of these effects while retaining the simplicity of layer models, some investigators introduce a cross-interface entrainment velocity that carries mass and momentum from one layer to the next. While quite common in studies of general circulation (e.g. Pedlosky 1996, chap. 3), this formulation has not been widely used in hydraulic theory. Gerdes, Garrett \& Farmer (2002) have discussed the consequences of an entrainment velocity for a single active layer. Fluid is allowed to enter the layer across its upper interface and it is assumed that the anomalous density and momentum are instantly mixed over the thickness of the layer. The active layer thus remains vertically homogeneous in density and horizontal velocity, although both quantities are allowed to vary in the along-channel direction. For the case in which the upper layer is motionless, it is shown that entrainment acts in a similar way to bottom friction, forcing the flow toward criticality and shifting the control point downstream from a sill or narrows. (These tendencies may change, however, if the upper layer is in motion.)

In reality, the mixing process associated with entrainment may not be complete. Fluid may be mixed over part of the water column, but not necessarily all the way to the bottom or instantaneously. In the Bab al Mandab, which is thought to contain significant mixing over its length, there exists smoothly varying density and velocity over the water column and no distinct interface (Murray \& Johns 1997; Pratt et al. 1999, 2000). Such observations call into question the use of layer models. The purpose of this work is to evaluate the performance of a layer (slab) model in the presence of entrainment and incomplete vertical mixing. We will concentrate on hydraulically driven flows, which differ from those broader flows normally associated with general ocean circulation in having much stronger inertial effects and more intense mixing.

Some insight into the questions raised above is provided by Garrett \& Gerdes (2003) who looked into the hydraulics of a homogeneous two-dimensional flow with vertical shear (and no entrainment). In the absence of friction, it is shown that critical flow occurs at the sill of an obstacle, as with a slab model. The critical condition itself can be expressed as an integral over the layer depth $d$ :

$$
\int_{h}^{h+d} \frac{g}{u^{2}} \mathrm{~d} z=1 .
$$


It can be shown from this formula that the depth average velocity $\bar{u}$ is $\geqslant(g d)^{1 / 2}$, so that the flow at the control section appears by traditional measure to be supercritical. The effects of bottom drag and internal friction are discussed by Garrett (2004) who argues that the critical condition is unchanged, but that the position of the control section is shifted to a point downstream of the sill. Bottom drag forces the control section downstream whereas internal friction does the opposite. In the limit of large viscosity, the internal flow is rendered slab-like and the control-section position is completely determined by bottom drag. In this case, the control section is predicted to lie where the bottom slope equals the negative of the quadratic drag coefficient, also the result obtained from a pure slab model (Pratt 1986). For weak internal friction the flow becomes strongly sheared and the effect of internal dissipation on the position of the control section becomes as strong as that of bottom drag. In this case, the control section is forced back upstream to near the sill. We will revisit this last effect in the discussion of our results.

To gain insight into the utility of the layer model with entrainment, we compare predictions based on this approach to numerical simulations based on a model with continuous stratification. We first review ( $\S 2$ and 3) the results of Gerdes et al. (2002) and show some examples constructed by applying their theory with the entrainment parameterization of Ellison \& Turner (1959). We next present a series of numerical results from the continuous model showing non-hydrostatic continuously stratified exchange flow over an obstacle $(\S 4)$. These flows are set up through a 'lock-exchange' experiment, configured to produce an overflow across the sill with a relatively inactive reverse flow above. (An independent barotropic flow can be added to increase the strength of the upper-layer flow.) Non-hydrostatic effects are allowed in order to study variations in the aspect ratio of the model, but most runs involve small ratios and are nearly hydrostatic. The critical section for the overflow is estimated (following Hogg, Ivey \& Winters 2001a) by introducing small-amplitude waves into the fluid at different locations and determining the point at which upstream propagation is cut off. We then attempt to fit the overflow to a layer representation by defining an upper interface that bounds the overflowing fluid. The shape of this interface and the location of the critical section are compared to the results obtained by integrating the equations of Gerdes et al. (2002) beginning with common upstream conditions. Although predicted trends are found in the continuous model, substantial quantitative disagreement is found $(\$ 5)$ between the two solutions in terms of the shape of the interface and the position of the control section.

We attempt to isolate the source of the disagreement between the continuous and layer model by reformulating the shallow-water equations, taking into account vertical variations of velocity and density within the active layer $(\S 6)$. The departure from the ordinary shallow-water equations is contained in three 'shape' parameters $\alpha, \beta$ and $\gamma$ that depend on the vertical profiles of horizontal velocity and density and that vary with the along-channel coordinate. These parameters appear as coefficients in the shallow-water equations for the vertically averaged properties of the active layer. It can be proved that $\alpha \geqslant 1$ and (for hydrostatically stable stratification) that $\beta \leqslant 1$, and these in turn imply that the flow tends to be more inertial than what would be indicated by the vertical mean velocity and density. Shallow-water theory is recovered when $\alpha=\beta=\gamma=1$, but it is shown that significant departures from this condition occur in the numerical simulations and in some geophysical applications ( 7 ). In other words, the effects of vertically varying density and horizontal velocity lead to important changes in the budgets for momentum and mass that make the flow more inertial than would be expected from ordinary shallow-water theory. In addition, 


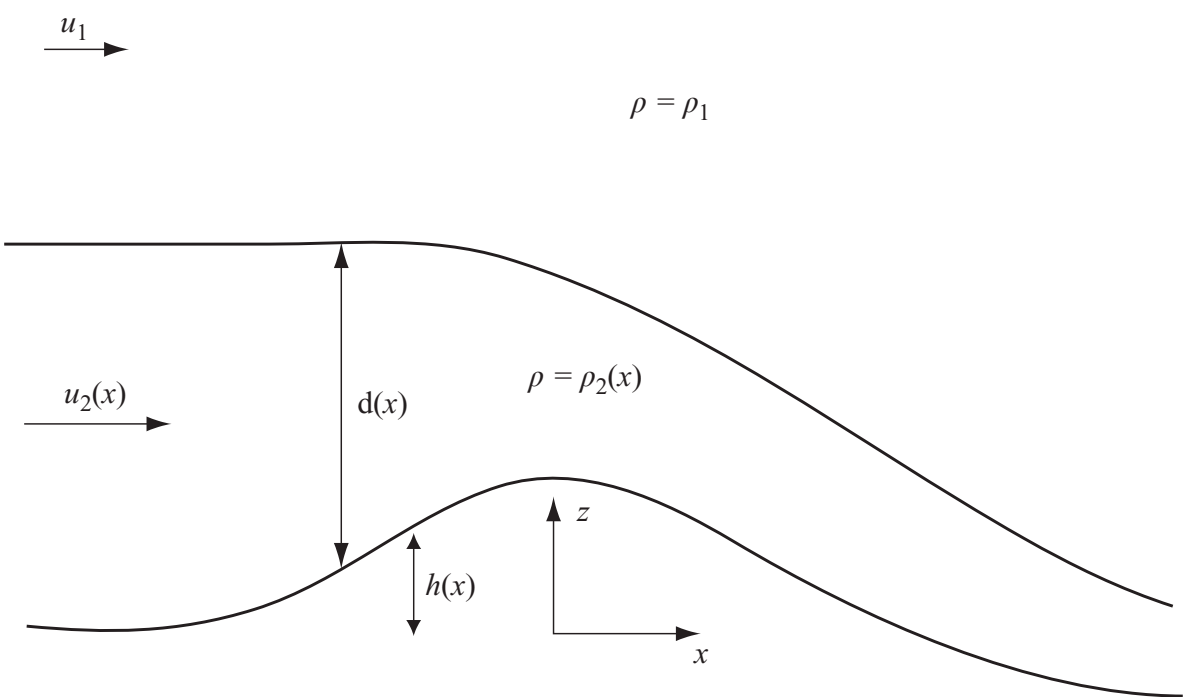

FIGURE 1. Definition sketch showing a deep homogeneous layer flowing beneath an inactive upper layer.

it is shown that under conditions of constant $\alpha, \beta$ and $\gamma$, the critical condition is $\bar{F}^{2}=\beta / \alpha \leqslant 1$, where $\bar{F}$ is the Froude number based on the depth-averaged flow. The section where this condition occurs lies upstream of the location at which $\bar{F}^{2}=1$. Constant $\alpha, \beta$ and $\gamma$ occur under conditions of self-similarity, which is not found in the simulations, but can occur under the conditions described by Wood (1968). As it turns out, the location predicted by this criterion lies quite close to the critical section identified in the full numerical simulations $(\S 6)$. The findings of Garrett (2004) suggest that agreement could be fortuitous, a consequence of internal frictional effects not present in the slab model.

\section{Governing equations for the layer system}

The Gerdes et al. (2002) formulation applies to a hydrostatic Boussinesq flow in a deep layer that is fed by entrainment from an overlying dynamically inactive upper layer (figure 1). The flow is confined to a channel with bottom elevation $z=h(x)$ and width that is assumed here to be constant. The upper layer (layer 1) may have a non-zero velocity $u_{1}$, but this velocity is spatially uniform and unaffected by changes in the lower layer. The entrainment process is represented by an (positive downwards) entrainment velocity $w_{e}$ that carries mass and momentum across the interface separating the layers. We consider cases of entrainment into the lower layer $\left(w_{e}>0\right)$, but not detrainment from the lower layer $\left(w_{e}<0\right)$. Momentum and mass carried across the interface are assumed to mix instantaneously over the depth of the lower layer, so that the lower-layer density $\rho_{2}$ and horizontal velocity $u_{2}$ remain vertically uniform. The lower-layer density will, however, vary horizontally, as will the value of reduced gravity $g^{\prime}(x)=g\left(\rho_{2}(x)-\rho_{1}\right) / \rho_{1}$.

Under these conditions, the evolution of the lower-layer velocity, thickness $(d)$, and Froude number $\left(F=u_{2} / \sqrt{g^{\prime} d}\right)$ are given by (Gerdes et al. 2002)

$$
\begin{aligned}
\frac{\mathrm{d} u_{2}}{\mathrm{~d} x} & =\frac{F^{2}}{\left(1-F^{2}\right) d}\left\{w_{e}\left(1+\frac{1}{2 F^{2}}-\frac{u_{1}}{u_{2}}\right)+\frac{u_{2}}{F^{2}} \frac{\mathrm{d} h}{\mathrm{~d} x}\right\}, \\
\frac{\mathrm{d}(d)}{\mathrm{d} x} & =\frac{F^{2}}{\left(1-F^{2}\right)}\left\{2 \frac{w_{e}}{u_{2}}\left(\frac{1}{4 F^{2}}+\frac{u_{1}}{2 u_{2}}-1\right)-\frac{1}{F^{2}} \frac{\mathrm{d} h}{\mathrm{~d} x}\right\},
\end{aligned}
$$




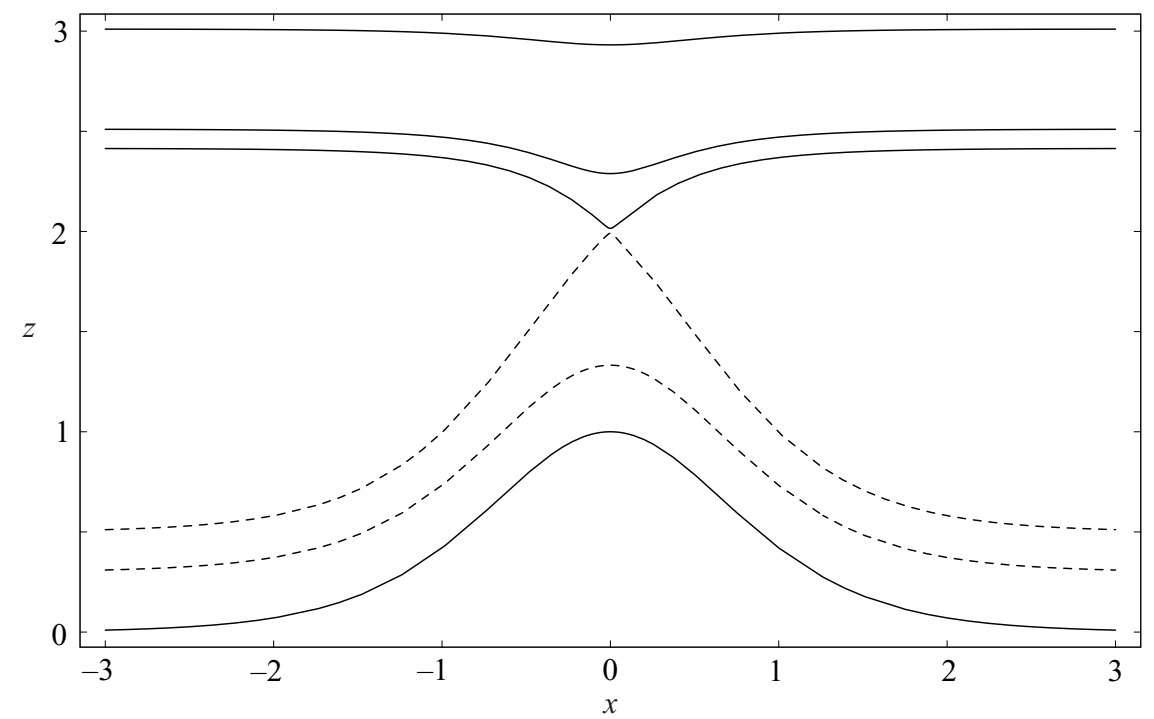

FiguRE 2. Solutions to (2.1)-(2.3) in terms of interface elevation for flow with $u_{1}=0, w_{e}=0$, $u_{2} d=1$ and with various upstream depths. Subcritical (supercritical) solutions are indicated by solid (dashed) curves. The topography consists of the single Gaussian shaped obstacle shown as the lowest curve. All quantities are non-dimensional.

and

$$
\frac{\mathrm{d} F^{2}}{\mathrm{~d} x}=\frac{3 F^{2}}{\left(1-F^{2}\right) d}\left\{\frac{w_{e}}{u_{2}}\left(F^{2}+\frac{1}{2}-\frac{u_{1}}{u_{2}} F^{2}\right)+\frac{\mathrm{d} h}{\mathrm{~d} x}\right\} .
$$

Let $h_{s}$ represent the obstacle height, $L$ the obstacle length, and $g_{0}^{\prime}$ the value of $g^{\prime}$ at some upstream section $x=x_{0}$. Then the above set of dimensional equations may be considered non-dimensional if the following replacements are made:

$$
\begin{gathered}
x \rightarrow x / L, \quad g^{\prime} \rightarrow g_{0}^{\prime}, \quad(d, h) \rightarrow(d, h) / h_{s}, \\
\left(u_{1}, u_{2}\right) \rightarrow\left(u_{1}, u_{2}\right) /\left(g_{0}^{\prime} h_{s}\right)^{1 / 2} ; w_{e} \rightarrow w_{e} L /\left(g_{0}^{\prime} h_{s}^{3}\right)^{1 / 2} .
\end{gathered}
$$

The non-dimensional version will be adopted hereinafter and therefore the obstacle height and length and the upstream value of $g^{\prime}$ will all be considered unity.

To illustrate some of the properties of solutions to the above, we will consider the flow over a simple obstacle (figure 1). The values $u_{2}\left(x_{0}\right)=u_{0}, d\left(x_{0}\right)=d_{0}$, and $F\left(x_{0}\right)=F_{0}$ are specified at an upstream location $x=x_{0}$ and (2.1)-(2.3) are integrated to find the downstream solution. If this is done for the case $w_{e}=0$, a set of standard hydraulic solutions are obtained (figure 2). The values of $u_{2} d, g^{\prime}(=1)$ and the Bernoulli function $u_{2}^{2} / 2+g^{\prime} d+g^{\prime} h$ are conserved for each solution. For the particular family of solutions shown, the volume flow rate per unit width $u_{0} d_{0}$ has been set to unity and $d_{0}$ is varied. For $d_{0}>2.41$, the solutions are completely subcritical, meaning that $u_{2}$ is everywhere less than the speed $\left(\sqrt{g^{\prime} d}\right)$ of long gravity waves. As shown in figure 2 , the interface elevation dips down and then rises as the fluid passes the obstacle. A family of supercritical solutions $\left(u_{2}>\sqrt{g^{\prime} d}\right)$ exists for $d_{0}<0.5$ and these experience a rise in interface elevation as the obstacle is passed. Also present are the hydraulically controlled solution $\left(d_{0}=2.41 \ldots\right)$, which is subcritical upstream and supercritical downstream of the obstacle, and its (unstable) mirror image for $d_{0}=0.5$. A more complete review of the properties of these solutions appears in Baines (1995).

When entrainment or bottom drag is present in the slab model, it is natural to ask whether $u_{2}-\left(g^{\prime} d\right)^{1 / 2}$ remains the wave speed for which hydraulic properties are judged. 
Although $u=\left(g^{\prime} d\right)^{1 / 2}$ would appear, on the basis of (2.1)-(2.3), to be the correct critical condition, it has not yet been shown that this corresponds to the presence of stationary waves. Moreover, Garrett \& Gerdes (2003) argue that the introduction of non-conservative processes in a shallow-water slab model gives rise to a family of dispersive long waves, some of which have upstream group velocity exceeding $u-\left(g^{\prime} d\right)^{1 / 2}$. This result would appear to cloud the traditional idea that upstream propagation cannot occur at a control section. Although their result is based on a model with quadratic bottom drag, the same issues are raised when entrainment is present.

These issues can be laid to rest by considering the full time-dependent equations for a shallow-layer subject to quadratic bottom drag and entrainment (Appendix A). Their characteristic form is

$$
\begin{gathered}
{\left[\frac{\partial}{\partial t}+\left(u_{2} \pm\left(g^{\prime} d\right)^{1 / 2}\right) \frac{\partial}{\partial x}\right]\left(u_{2} \pm 2\left(g^{\prime} d\right)^{1 / 2}\right) \mp\left(\frac{d}{g^{\prime}}\right)^{1 / 2}\left[\frac{\partial}{\partial t}+\left(u_{2} \pm\left(g^{\prime} d\right)^{1 / 2}\right) \frac{\partial}{\partial x}\right] g^{\prime}} \\
=-g^{\prime} \frac{\mathrm{d} h}{\mathrm{~d} x}-C_{d} \frac{u_{2}\left|u_{2}\right|}{d}+w_{e}\left[\frac{u_{1}-u_{2}}{d}+\frac{g^{\prime}}{2 u_{2}} \pm\left(\frac{g^{\prime}}{d}\right)^{1 / 2}\right]
\end{gathered}
$$

and

$$
\left[\frac{\partial}{\partial t}+u_{2} \frac{\partial}{\partial x}\right] g^{\prime}=-\frac{g^{\prime} w_{e}}{d},
$$

where $C_{d}$ is the bottom drag coefficient. There are three characteristic speeds: $u_{2} \pm\left(g^{\prime} d\right)^{1 / 2}$ and $u_{2}$, and a sketch of the corresponding characteristic curves for a hypothetical steady flow over an obstacle appears in figure 3. These curves represent the paths that small-amplitude perturbations of the steady flow would take in the $(x, t)$-plane. The dark solid curves correspond to signals with speed $u_{2}-\left(g^{\prime} d\right)^{1 / 2}$, while the dashed and faint solid curves correspond to speeds $u_{2}+\left(g^{\prime} d\right)^{1 / 2}$, and $u_{2}$. The latter two have positive tilt everywhere, corresponding to downstream propagation of information. It is assumed that $u_{2}=\left(g^{\prime} d\right)^{1 / 2}$ at a location slightly downstream of the sill and this is represented by a vertical solid curve in the $(x, t)$-plane. Upstream propagation is possible only to the left of this line.

The above formulation is made possible because bottom drag and entrainment do not involve $x$ - or $t$-derivatives of the dependent variables. Under these conditions, information propagates along characteristic curves that are defined in the same way as if the flow were conservative. The effect of non-conservation is that the signals themselves are modified as they propagate. Even so, the solution at any location upstream of the sill is influenced only by information that exists upstream of the sill. For example, the solution at location $B$ (figure 3 ) is influenced only by initial conditions lying along $A C$. It would appear then that $u_{2} \pm\left(g^{\prime} d\right)^{1 / 2}$ and $u_{2}$ are the only signals speeds relevant to the hydraulics of the flow and that apparent dispersive behaviour as identified by Garrett \& Gerdes (2003) is really a manifestation of the alteration of information along the signal paths by entrainment, bottom topography or, in their case, bottom friction.

A related principle that can guide the selection of wavelength relevant to hydraulic criticality is that of non-dispersion. When forced locally, a stationary non-dispersive wave is unable to transport energy away from the site of forcing, resulting in resonance. This process is evident in the right-hand sides of (2.1)-(2.3), which indicate resonant excitation when the speed $u_{2}-\left(g^{\prime} d\right)^{1 / 2}$ of a non-dispersive wave becomes zero. Resonant growth is avoided only when the sum of the forcing terms (the numerators) 


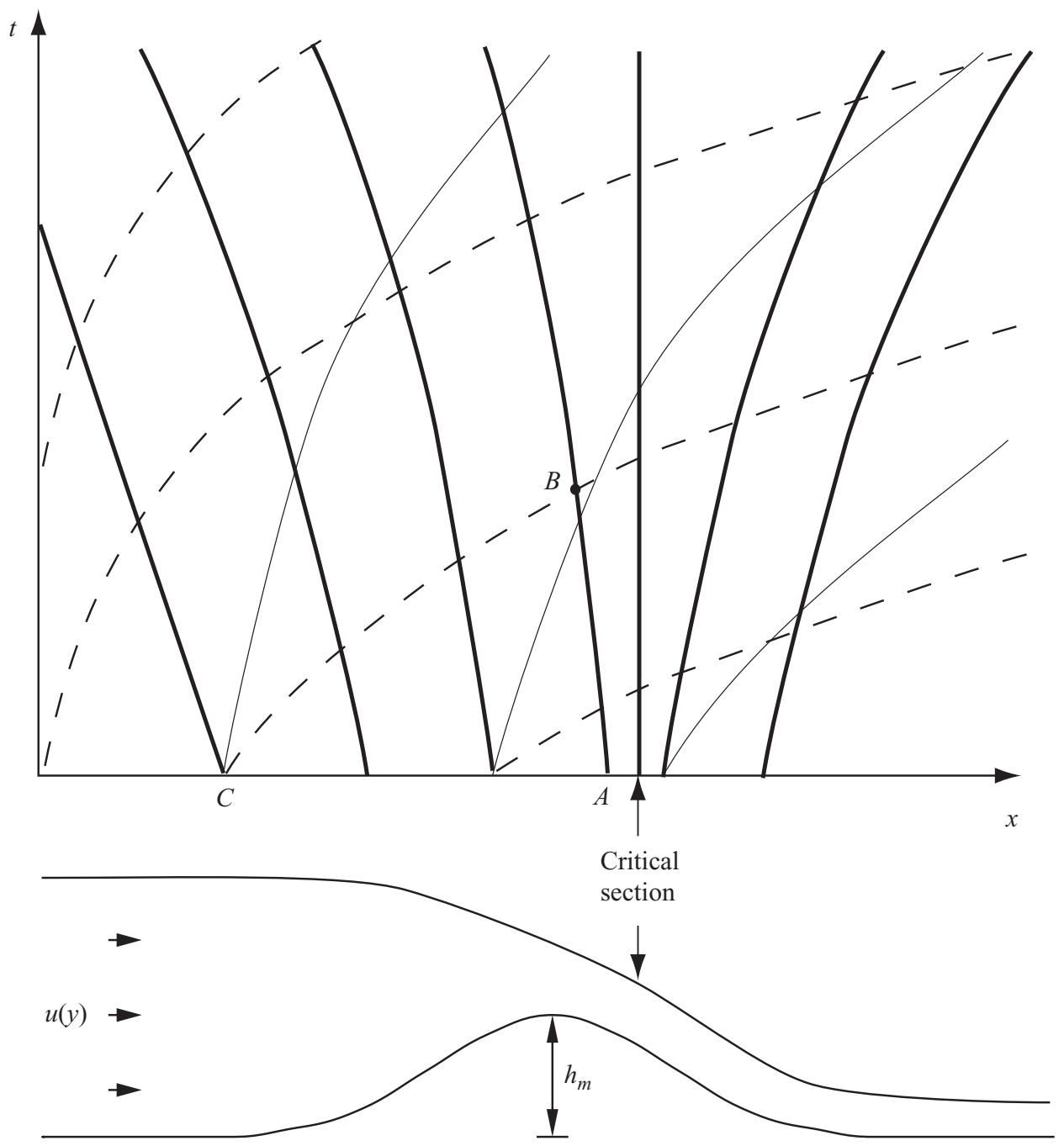

FiguRE 3. The lower frame shows a hydraulically controlled flow in which entrainment and/or bottom drag have caused the critical section to be located downstream of the sill. The upper frame shows the characteristic curves for the steady flow, also the paths in the $(x, t)$-plane along which small amplitude disturbances to the steady flow would travel. The characteristic speeds are given by $u_{2}-\left(g^{\prime} d\right)^{1 / 2}$ (thick, solid curves) $u_{2}+\left(g^{\prime} d\right)^{1 / 2}$ (dashed) and $u_{2}$ (thin, solid).

add up to zero:

$$
\frac{w_{e}}{u_{2}}\left(\frac{3}{2}-\frac{u_{1}}{u_{2}}\right)=-\frac{\mathrm{d} h}{\mathrm{~d} x} \quad\left(F^{2}=1\right),
$$

which follows from any of (2.1)-(2.3). If $w_{e}=0$, then the only momentum source is the horizontal component of the bottom pressure, here $\mathrm{d} h / \mathrm{d} x$. Critical flow must occur where this slope is zero, at the sill in figure 1. Entrainment provides a source of mass and of momentum (if $u_{1} \neq u_{2}$ ) to the lower layer. If $u_{1}$ is non-positive, critical flow must occur on the downslope $\mathrm{d} h / \mathrm{d} x<0$ of the obstacle. Critical flow can occur on the upslope $\mathrm{d} h / \mathrm{d} x>0$ of the obstacle if the upper-layer velocity is positive and sufficiently strong $\left(u_{1}>3 u_{2} / 2\right)$. If the magnitude of the left-hand side of (2.6) is everywhere larger than the maximum bottom slope, then critical flow will be completely expunged from the problem and no hydraulic transitions will occur. (Hogg et al. (2001a) have shown that hydraulic behaviour is expunged from a viscous, stratified, exchange flow for sufficiently small values of the parameter $g^{\prime} h_{s}^{5} / \nu^{2} L^{2}$ where 
$v$ is the vertical eddy vicosity. If we interpret $\sqrt{g^{\prime} h_{s}}$ as a scale for velocity an $v / h_{s}$ as a scale for $w_{e}$ then the condition (2.6) leads to a similar conclusion.)

\section{Examples of solutions with Ellison \& Turner entrainment}

A standard parameterization for the entrainment velocity is that due to Ellison \& Turner (1959, hereinafter referred to as ET). In the present non-dimensional units, $w_{e}$ is given by

$$
w_{e}= \begin{cases}\left|u_{1}-u_{2}\right| \frac{L}{h_{s}}\left(\frac{0.08-0.1 R_{i}}{1+5 R_{i}}\right) & \left(R_{i}<0.8\right) \\ 0 & \left(R_{i} \geqslant 0.8\right)\end{cases}
$$

where

$$
R_{i}=F^{-2}\left(\frac{u_{1}}{u_{2}}-1\right)^{-2}
$$

If the upper layer is motionless $\left(u_{1}=0\right)$ then $F=R_{i}^{-2}$ and the requirement $R_{i}<0.8$ means that entrainment only occurs for supercritical flows.

Use of the ET expression for $w_{e}$ in (2.6) leads to a more specific constraint on the position of a critical section:

$$
\frac{\mathrm{d} h}{\mathrm{~d} x}= \begin{cases}-\left|\frac{u_{1}}{u_{2}}-1\right|\left(\frac{3}{2}-\frac{u_{1}}{u_{2}}\right) \frac{L}{h_{s}}\left[\frac{0.08\left(u_{1} / u_{2}-1\right)^{2}-0.1}{\left(u_{1} / u_{2}-1\right)^{2}+5.0}\right] & \left(R_{i}<0.8\right), \\ 0 & \left(R_{i} \geqslant 0.8\right) .\end{cases}
$$

According to (3.2) (with $F=1$ ), the condition $R_{i} \geqslant 0.8$ is satisfied if $u_{1} / u_{2}>2.12$ or if $u_{1} / u_{2}<-0.12$. In the first case, the upper layer flows in the same direction and at a greater speed than the lower-layer critical flow, and the critical section lies on the upslope of the obstacle. In the second case, the upper-layer velocity is negative and the critical section lies on the downslope of the obstacle.

It is interesting to observe how the conservative solutions of figure 2 are altered when ET entrainment is introduced. To this end we have calculated a family of solutions by fixing the upstream $\left(x_{0}=-3\right)$ values of $u_{2} d$ and of $g^{\prime}$ at unity and varying the upstream value of $d$, as before. The value of $L / h_{s}$ is fixed at 5 and solutions are obtained by integrating the dimensionless versions of (2.1)-(2.3) from $x=-3$ in the direction of positive $x$. When the upper layer is motionless $\left(u_{1}=0\right)$ the subcritical (solid curves) solutions remain unchanged from the previous case, as shown by the curves of interface elevation (figure $4 a$ ) and Froude number (figure $4 b$ ). On the other hand, the supercritical (dashed) solutions such as the one with $d(-3)=0.05$ experience rapid increases in depth owing to entrainment.

Critical flow at the sill is obtained when the upstream flow is subcritical and has value $d(-3) \cong 2.41$ or when the upstream flow is supercritical and has value $d(-3) \cong 0.26$. In each case, the subcritical and supercritical branches of the solution that occur downstream of the critical section are shown. The appropriate choice of downstream solution is the one that allows the fluid to pass smoothly through the critical section. For example, we would follow the subcritical (solid) curve beginning at $d(-3) \cong 2.41$ and continue on to the supercritical (dashed) branch downstream of the sill. The subcritical downstream branch has an upstream continuation with values $u_{2} d$ and $g^{\prime}$ different from those used to generate the family of curves in figure $3(a)$. This solution is unstable, as is the supercritical-to-subcritical solution with $d(-3) \cong 0.26$. 
(a)
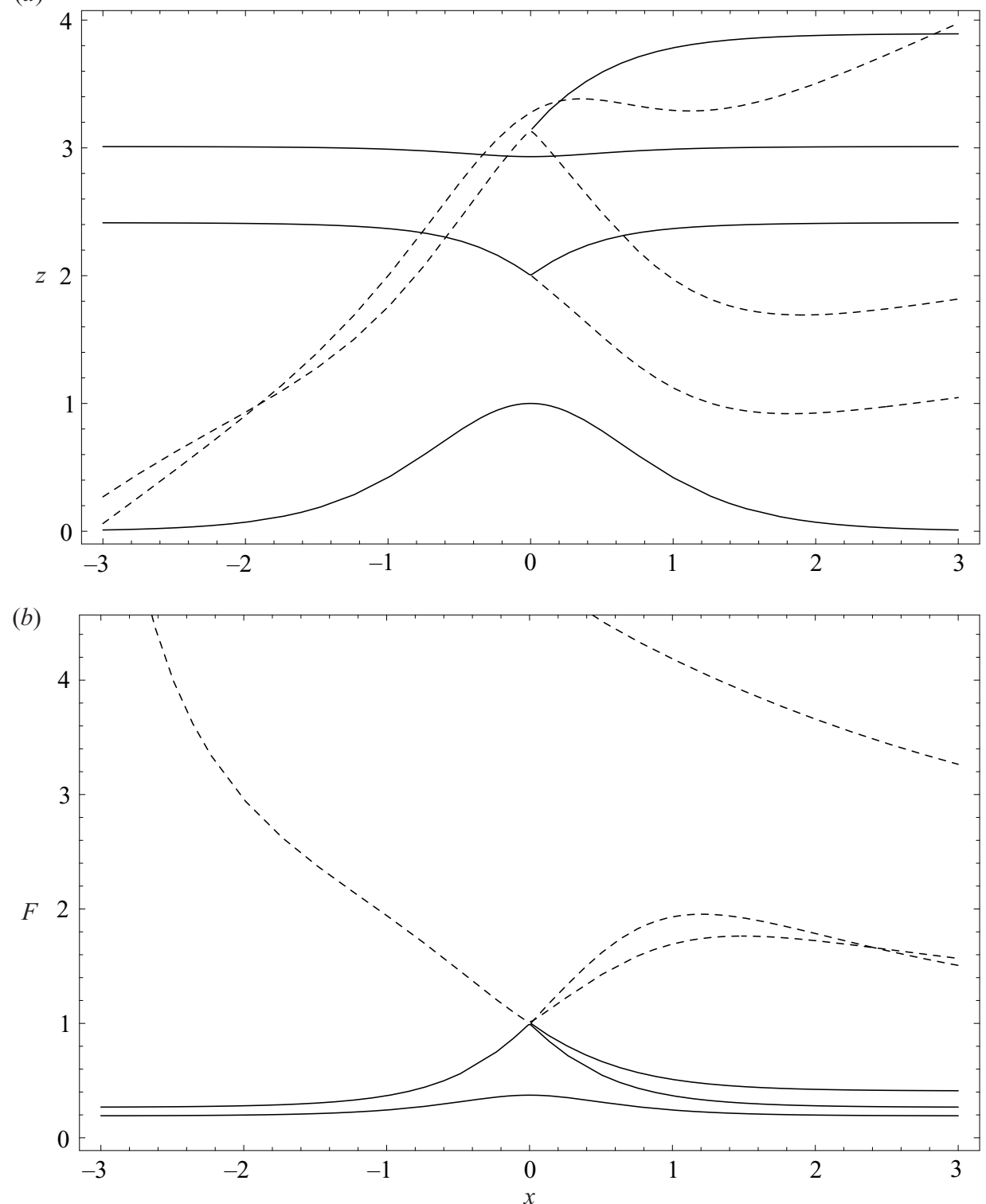

FIgURE 4. (a) Same as figure 2 except that $w_{e}$ is given by (3.1) with $L / h_{s}=5$. (b) The Froude numbers $F=\left(g^{\prime} d\right)^{1 / 2}$ corresponding to the solutions shown in $(a)$.

Intersections between different solution curves in figure $4(a)$ do not carry the same significance as would be the case in a conservative system. In the latter, intersections imply the existence of two solutions with the same depth and fluxes, but different interface slopes. Such behaviour is indicative of critical flow since it implies that stationary disturbances can exist at the section in question. An example is the intersection point corresponding to critical sill flow in figure 2. For the (nonconservative) solutions shown in figure 4(a), an intersection implies only that the depths of the two solutions are equal, not necessarily the fluxes or values of $g^{\prime}$. For example, the intersection between the dashed curves near $x=-1.9$ involves two solutions with identical depths but different Froude numbers (as shown in the figure $4 b$ ).

As pointed out by Gerdes et al. (2002), entrainment that occurs in the presence of non-positive $u_{1}$ tends to push the flows towards criticality. This property can be 

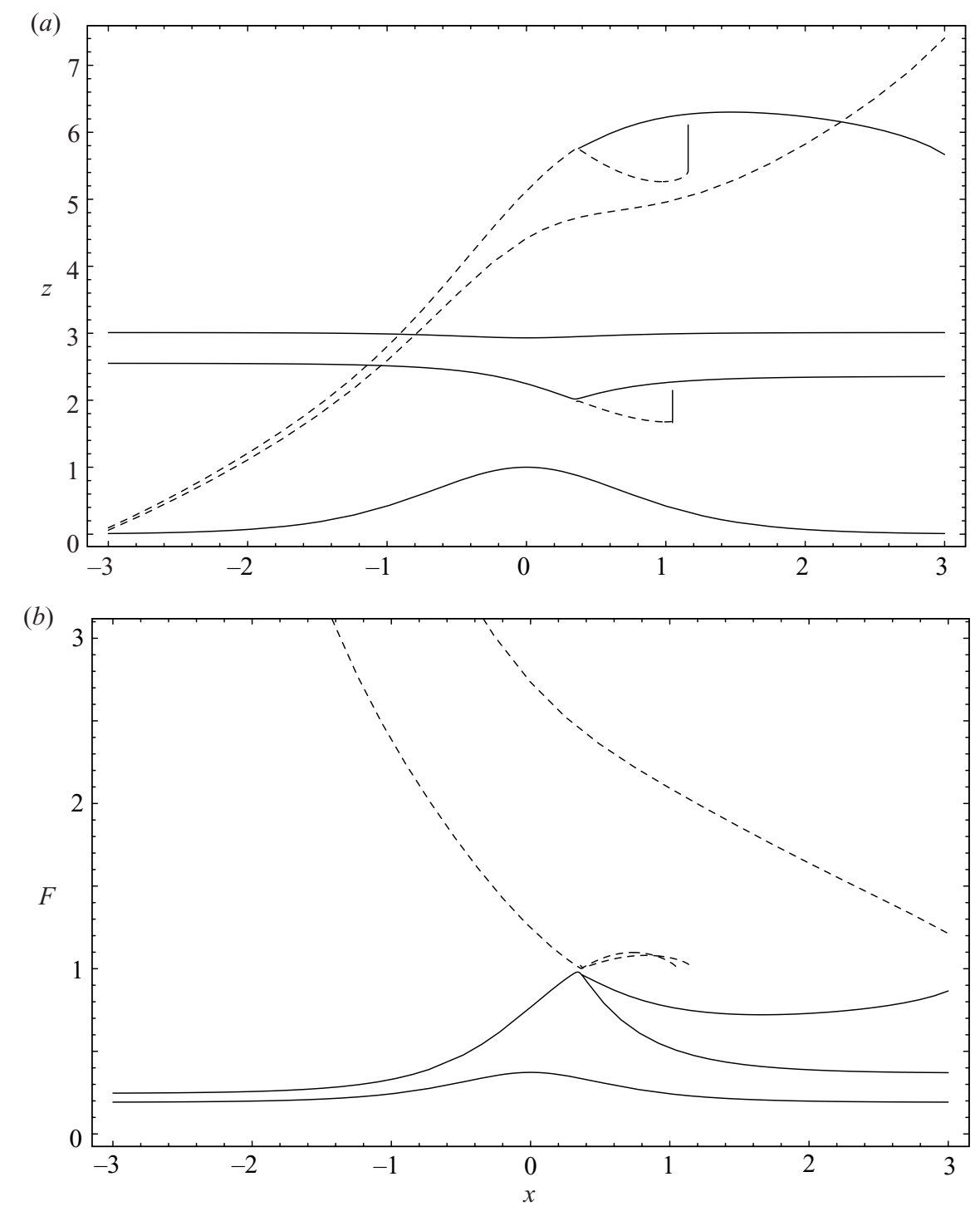

FIGURE 5. As figure 4 except that $u_{1}=-1$.

seen by inspection of (2.3), which shows that $\partial F^{2} / \partial x<0(>0)$ when $F^{2}>1(<1)$, not accounting for the influence of topography and provided $u_{1} / u_{2} \leqslant 0$. This behaviour is confirmed by the Froude number behaviour of the solutions (figure $4 b$ ).

The previous case has $u_{1}=0$, so entrainment occurs only when the flow is supercritical. An important consequence is that critical flow can occur only at the sill. We now consider two cases with finite upper velocity, $u_{1}=-1$ (figure 5) and $u_{1}=3$ (figure 6). Inspection of figure 5(a) shows that critical transitions occur downstream of the sill as predicted by (3.3). As in the previous case, entrainment tends to push the solutions towards a critical state (figure $5 b$ ) and, in the case of some of the supercritical curves, this results in the formation of an infinite interface slope corresponding to a hydraulic jump. Jumps are represented in the figure by vertical terminations of the dashed curves.

The case of $u_{1}>0$ (figure 6) is less straightforward because it is more difficult to generalize the tendency for entrainment to push the flow towards or away from criticality. According to (2.3), the tendency is to force the flow away from the critical 
(a)
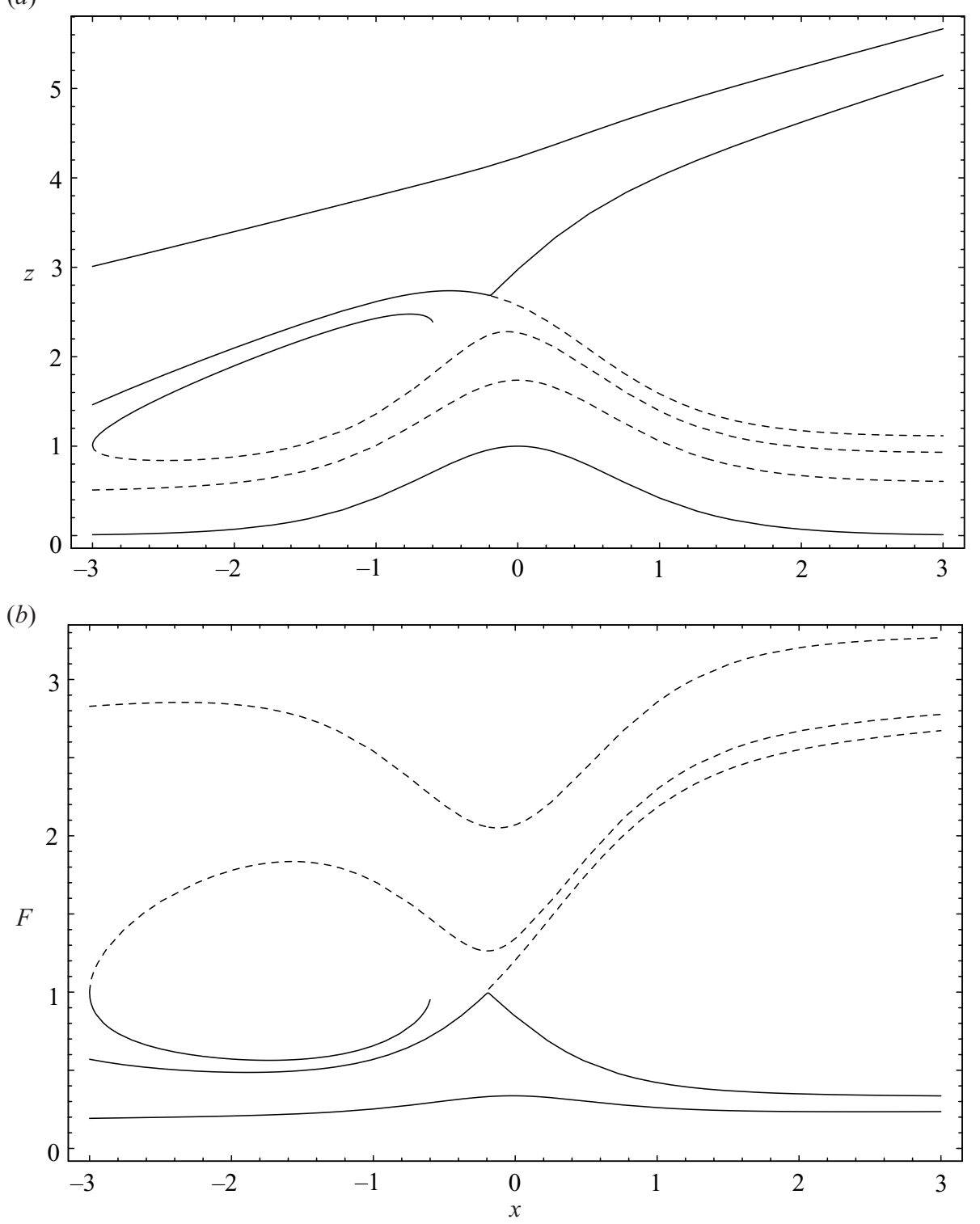

FIGURE 6. As figure 4 except that $u_{1}=3$.

state if $u_{1}$ is sufficiently large that

$$
\left(1-u_{1} / u_{2}\right) F^{2}<-\frac{1}{2}
$$

Also, (2.6) shows that displacement of the critical section upstream requires $u_{1} / u_{2}>3 / 2$. The solutions shown in figure 6 were computed with a strong upper layer velocity $\left(u_{2}=3\right)$ resulting in satisfaction of both conditions. The traditional, hydraulically controlled solution begins with upstream depth $\cong 1.5$ and remains subcritical until $x \cong-0.2$ where a transition to supercritical flow occurs (figure $6 a$ ). As it turns out, there is another control section right at the upstream boundary $x=-3$ where the depth is $\cong 1$. Sub- and supercritical solution curves branch from this point, but only the supercritical branch is continuous across the entire obstacle. The Froude number curves of figure 6(b) show a tendency for solutions to move away from criticality, except where topography may reverse this trend. 


\section{Solutions based on a numerical model with continuous stratification}

To what extent do the solutions of the previous section reproduce the characteristics of continuously stratified deep overflows when the vertical mixing of entrained fluid is incomplete? We seek a limited answer to this question by performing a set of numerical experiments based on the incompressible Boussinesq equations in two dimensions:

$$
\begin{gathered}
\frac{\partial \boldsymbol{U}}{\partial t}+(\boldsymbol{U} \cdot \nabla) \boldsymbol{U}=-\frac{1}{\rho_{0}} \nabla p^{\prime}-\boldsymbol{g}^{\prime}+\frac{1}{\rho_{0}} \nabla \cdot\left(K_{m} \nabla \boldsymbol{U}\right), \\
\frac{\partial g^{\prime}}{\partial t}+\boldsymbol{U} \cdot \nabla g^{\prime}=\nabla \cdot\left(K_{s} \nabla g^{\prime}\right),
\end{gathered}
$$

and

$$
\nabla \cdot \boldsymbol{U}=0
$$

in which $\boldsymbol{U}$ is the velocity vector, $\rho_{0}$ is the reference density, and $g^{\prime}$ and $p^{\prime}$ are the perturbation quantities of the density and the pressure, respectively, all variables according to the non-dimensionalization introduced in $\S 2 . K_{m}$ and $K_{s}$ are the diffusion coefficients for momentum and density, respectively. The numerical scheme used to solve these equations is the second-order projection method described by Bell et al. $(1989 a, b)$ and Bell \& Marcus (1992). Essentially, the projection is to estimate $\boldsymbol{U}$ at the new time step $n+1$ using the pressure gradient calculated from the preceding half time step $n-1 / 2$, i.e. the pressure gradient is taken as a source term in (4.1). This estimate for $\boldsymbol{U}$ will be divergent, and so the non-divergent part, obtained as the curl of $\boldsymbol{U}$, is removed. This part is then used to find the perturbation pressure gradient at the new half time step $n+1 / 2$. The diffusion coefficients $K_{m}$ and $K_{s}$ are calculated according to Smagorinsky (1963). The Smagorinsky constant, which is used to calculate $K_{m}$ from the computed velocity and density gradients, is taken to be 0.17 (Winters \& Seim 2000). Also following Winters \& Seim, the turbulent Prandtl number is taken as unity, and so $K_{m}=K_{s}$.

The model domain is bounded by rigid impermeable walls at the top and bottom and has open boundaries at the ends. The top is horizontal, and the bottom includes a topographic obstacle described by

$$
h=\exp \left(-\left(\frac{L}{h_{s}} x\right)^{2}\right),
$$

in which $h$ is scaled by $h_{s}, x$ by $L$, and where $h_{s} / L$ is the obstacle aspect ratio. At the top and the bottom we operate with a slip velocity, in accordance with the neglect of surface drag in (2.1)-(2.3). The computational domain consists of an orthogonal curvilinear grid following the topography. It is created from the model domain by the method described by Ives \& Zacharias (1987). This method conformally maps grid points designated on the boundaries of the model domain (in Cartesian coordinates) onto a rectangle. Then, the interior points of the grid are calculated using a Poisson solver technique. For the present purpose, the grid points on the vertical boundaries are distributed so that a high density of grid points is obtained in the lower part of the physical domain, which is where the active layer of fluid is found. Along the top and bottom boundary, the grid points are distributed with constant spacing.

We have used different computational domains, all with 50 grid points in the vertical and 1000 grid points in the horizontal, but with different lengths and heights 
of the obstacle. In every case, the total length of the domain was 10 times or more the length of the obstacle. The height of the obstacle was either 0.05 or 0.1 of the total height of the domain. With lower-layer depths up to twice the obstacle height, we have kept the lower layer approximately decoupled from the upper layer.

The initial conditions for all our model runs consist of a two-layer stratification throughout the model domain. To the left of the obstacle, the lower-layer depth is set to twice the obstacle height, and to the right the lower layer is kept slightly below the crest of the obstacle. Further, both fluids are stagnant initially, and so these experiments resemble a dam-break problem. In case of inflow through the open boundaries (resulting from adjustments within the model domain or a barotropic flow through it), the densities assigned initially at these places are used. Sponge layers are also applied at both ends in order to damp reflections from the open boundaries. Finally, we control the barotropic flow component by prescribing the value of the streamfunction on the upper boundary.

All model results presented are of flows that have developed to steady state and are smooth with no turbulence. In times immediately after initiation of the flow, however, overturning Kelvin-Helmholtz billows are present and resolved. These billows are subsequently dissipated by the mixing scheme, leaving the smooth steady flow. The choice of the non-hydrostatic model and the mixing scheme was made partly for convenience, but also to maintain a connection with the modelling of Hogg, Winters \& Ivey (2001b). However, the choice of model is not crucial. Any model that produces incomplete mixing of density and momentum through the lower layer and from which the vertical entrainment can be determined is adequate to test the central ideas. Whether the model produces more (or less) mixing than would occur in a laboratory or oceanographic flow is of secondary importance. As it turns out, the redistribution of momentum and velocity as a result of mixing produced in the model is qualitatively similar to the distributions observed in the Romanche fracture zone overflow ( $\$ 7)$, but this is not by design.

The model is allowed to run roughly until the disturbances from the initial dam break have reached the ends of the domain. At this time, the flow becomes nearly steady in the vicinity of the obstacle. Three different obstacle aspect ratios are used $\left(h_{s} / L=1.41 \times 10^{-1}, 3.54 \times 10^{-2}\right.$ and $\left.1.77 \times 10^{-2}\right)$ and the upper-layer velocity is varied within each case by prescribing the barotropic flow. The main restriction on the barotropic component is that it should not be so large as to make the upper layer dynamically active. We impose this restriction by requiring that the upper-layer Froude number must remain much smaller than the lowerlayer Froude number. By performing a suite of experiments for each $h_{s} / L$, we map out a parameter space in which the general character of the final flow can be categorized. One quantity of particular interest is the location of a critical section.

Consider an example of the model output for a run with an aspect ratio $h_{s} / L=1.41 \times 10^{-1}$, a domain height of $20 h_{s}$ and with zero barotropic flow (figure 7). The overall pattern consists of a positive lower level flow that spills over the obstacle. The overflow underlies a reverse flow with a velocity $u_{1}$ that is everywhere much less in magnitude than $u_{2}$. Entrainment occurs where the lower layer accelerates and flows over the obstacle, as shown in the pattern of subducted streamlines (figure $7 c$ ). The effect of the entrainment on the distributions of density and velocity can be seen in figures $7(a)$ and $7(b)$, respectively. For the density, the interfacial area between the 

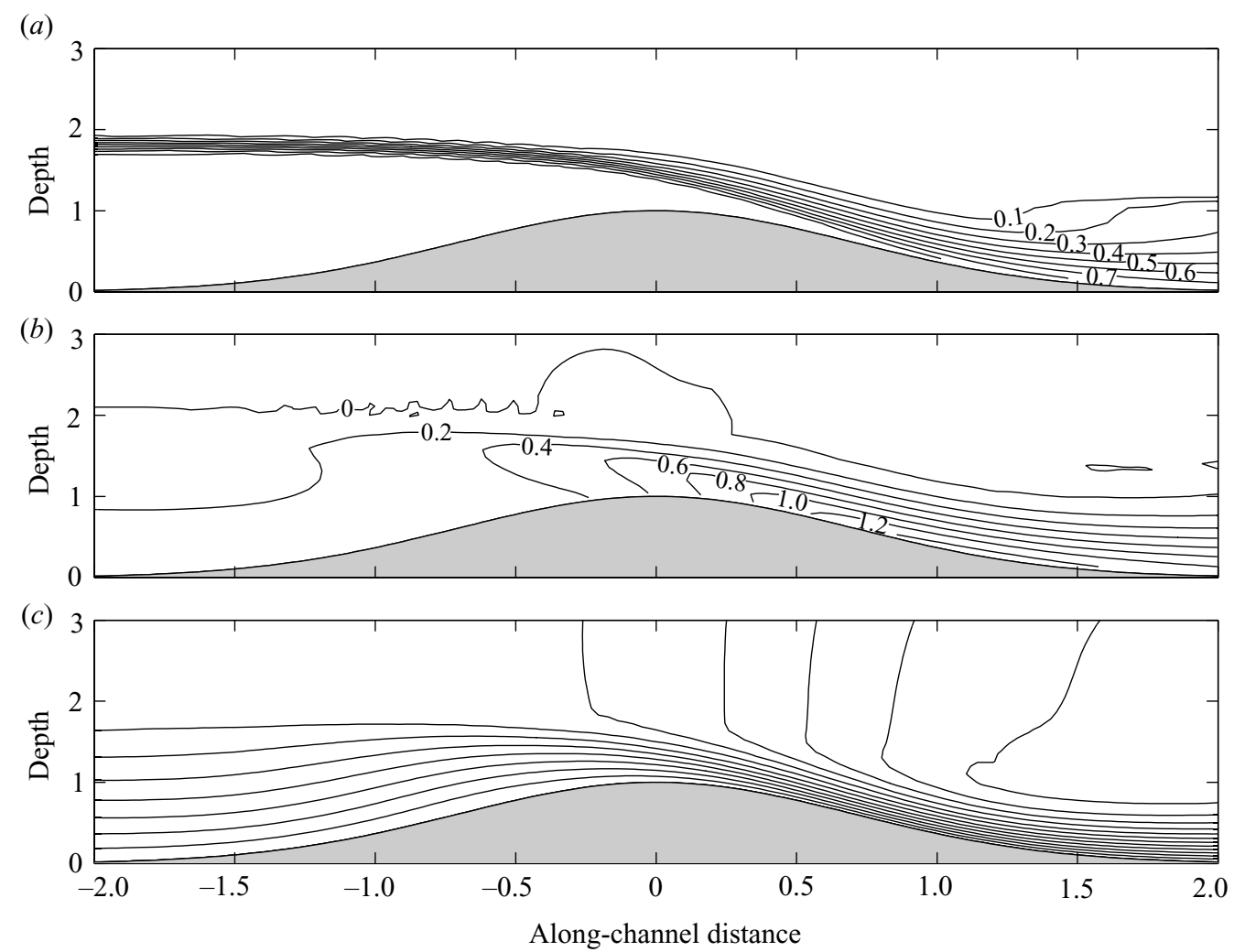

FIGURE 7. Output of the non-hydrostatic model run for an obstacle aspect ratio at $1.41 \times 10^{-1}$ and an upper-layer flow at almost zero. (a) Contours of density anomaly between the upper and the lower layer; $(b)$ contours of speed; $(c)$ the streamlines. All units are non-dimensional according to $\S 2$.

two homogeneous layers increases gradually and ends up extending all the way to the bottom. For the velocity, as the flow approaches the obstacle, slightly higher values are found in the upper part of the lower layer. This seems to change as entrainment increases; the highest velocities gradually being found near the bottom. Note that the lower layer is not at all homogeneous in density and velocity as entrainment takes place.

In order to diagnose the model output in the frame of reference provided by (2.1)(2.3), a layered structure is defined. The interface is defined as the location of the isopycnal $g^{\prime}=0.01$ (in the non-dimensional formulation), which is close to the density of the upper layer, and so practically all parts of the fluid that have undergone mixing will be considered as belonging to the lower layer. We also attempted to define an interface based on the level of zero horizontal velocity, but the resulting contour was quite irregular. Having defined the interface, the mean velocity $\bar{u}_{2}$, the mean reduced gravity $\bar{g}^{\prime}$, and the Froude number $\bar{F}$ for the lower layer are calculated as follows:

$$
\begin{aligned}
& \bar{u}_{2}=\frac{1}{d} \int_{h}^{h+d} u_{2} \mathrm{~d} z \\
& \bar{g}^{\prime}=\frac{1}{d} \int_{h}^{h+d} g^{\prime} \mathrm{d} z
\end{aligned}
$$



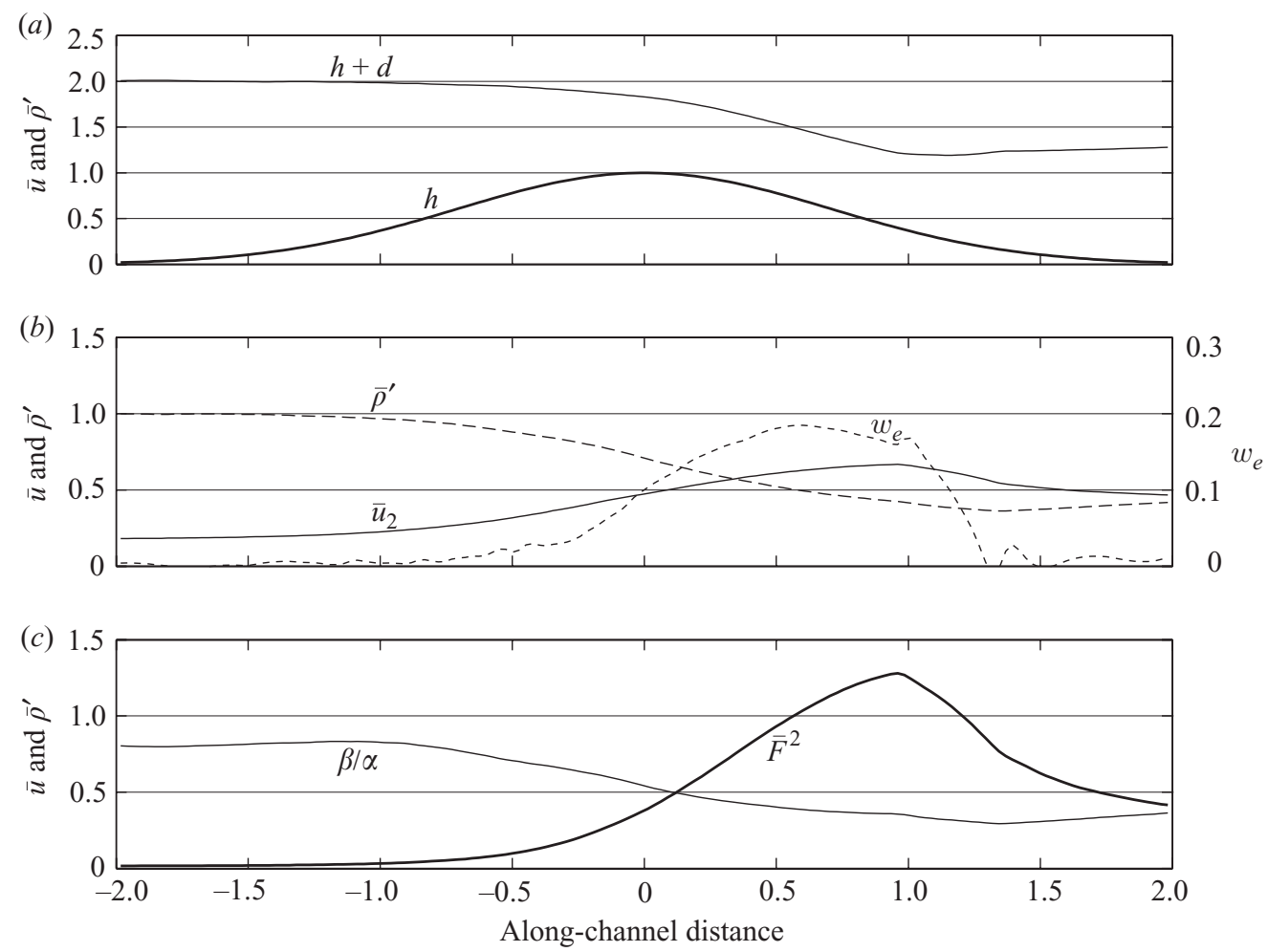

FiguRE 8. A layered interpretation of the model output shown in figure 7. (a) The height of the obstacle (thick line) and the interface elevation (thin line); $(b)$ mean velocity, $\bar{u}_{2}$ (solid line), the mean density anomaly, $\bar{\rho}^{\prime}$ (long dashes), and the entrainment velocity, $w_{e}$ (short dashes use axis on the right); (c) the Froude number, $\bar{F}^{2}=\bar{u}_{2}^{2} /\left(\bar{g}^{\prime} d\right)$ (thick line), and $\beta / \alpha$ (solid line), defined in $\S 6$.

and

$$
\bar{F}^{2}=\frac{\bar{u}_{2}^{2}}{\bar{g}^{\prime} d} .
$$

The entrainment velocity $\left(w_{e}\right)$ is calculated using conservation of volume for the lower layer and it is verified that the result closely approximates to the vertical velocity across the interface produced by the model. When determining the location of the interface, noise arises in the along-channel direction owing to the discrete representation of the density field. This noise is removed by applying a moving average to the variables pertaining to the layered description.

The interface elevation and mean velocity and density obtained using the above procedure with the figure 7 model run are shown in figure 8. A good measure for the degree of mixing that is taking place is the mean density, non-dimensionalized by its value at $x=-2.0$ and shown in the middle panel in the form $\bar{g}^{\prime}$. The latter is seen to decrease by more than $50 \%$ over the region where $w_{e}$ is largest (also shown figure $8 b$ ), implying a decrease by more than a factor of 2 in the mean layer density. The Froude number $\bar{F}$ based on the mean flow (figure $8 c$ ) reaches the value unity at $x=0.56$.

To estimate the location of the actual control point for each model run, a number of wave excitation experiments were carried out. A small-amplitude approximately hydrostatic disturbance was introduced in different locations near the crest of the obstacle by raising the isopycnals a small amount. The disturbance had the shape of 
one sine wave and was fitted smoothly to the steady solution. The bottom and rigid lid pressures were adjusted to eliminate any barotropic component (which would travel upstream at an infinite speed). Then, the model was run forward and the resulting wave-propagation pattern was found by subtracting the undisturbed flow field. The purpose of the experiments is to determine the section at which small-amplitude long waves remain stationary. The procedure is similar to that used by Hogg et al. (2001b) who calculated the evolution of a free disturbance using linearized versions of the governing equations.

Interpretation of the results of this approach is subject to several sources of uncertainty. As noted by Hogg et al. (2001b), the gravest wave modes of the flow can generally be classified as internal or 'vortical'. The former have the strongest effect on stratification and are therefore of primary interest here. Vortical modes can, in principle, propagate upstream through a critical section for internal waves, these waves apparently cause no alteration of the upstream stratification. The waves we introduce involve disturbances of the stratification, but not the vorticity, thereby minimizing the generation of vortical modes. A second complication is the presence of friction and buoyancy diffusion, which can lead to dispersion and strong damping as the wavelength goes to infinity. Our guiding principle is that the waves associated with hydraulic control should be non-dispersive, and we therefore select wavelengths sufficiently long to be hydrostatic, but shorter than the characteristic scale for which damping becomes important. It is difficult to determine the precise time or space scale for any damping of the disturbance. However, following Hogg et al. (2001b), the relative effects of advection versus diffusion can be estimated by comparing the ratio of the time for the wave to propagate one wavelength to the time for momentum to diffuse vertically over the depth of the active layer

$$
t_{a} / t_{b}=K_{m} / k,
$$

where the turbulent diffusion coefficient $K_{m}$ has been normalized by $\left(g^{\prime} h_{s}^{3}\right)^{1 / 2}$ and the disturbance wavelength $k$ by $h_{s}$. They found that their results were not sensitive to the viscosity for $t_{a} / t_{d}<10^{-2}$. For the calculations presented below (figure 10), $K_{m}=1.5 \times 10^{-3}$ in the neighbourhood of the sill crest and the primary wavelength of the truncated sine wave disturbance $k=1.77$, giving $t_{a} / t_{d}=8 \times 10^{-4}$, suggesting that damping of the disturbance is probably insignificant.

Figure 9 shows the temporal evolution of the bottom velocity $u(x, h)$ resulting from disturbances introduced at $x=0$ (at the crest of the obstacle), 0.14, 0.28, 0.42 and 0.56. In each case, the disturbance develops into two parts, one of negative velocities (relative to the steady solution) on the upstream side and one of positive velocities on the downstream side. When the wave is excited at $x=0$, it appears to be able to propagate in both the upstream and the downstream direction. This suggests that the flow is subcritical at $x=0$. When excited at $x=0.28$, or downstream, propagation takes place in the downstream direction only, indicating that the flow regime is supercritical in this area. At $x=0.14$, the phase speed of the part of the wave to the upstream side seems close to zero, showing that the control point is close to this location. Evidently, the place of control is much farther upstream than where $\bar{F}^{2}=1(x=0.56$, as shown in figure 8$)$.

\section{Comparison between the continuous and shallow-water models}

How well does the layer model capture the dynamics of the deep overflows seen in the numerical simulations? We now investigate this issue in the context of the steady 


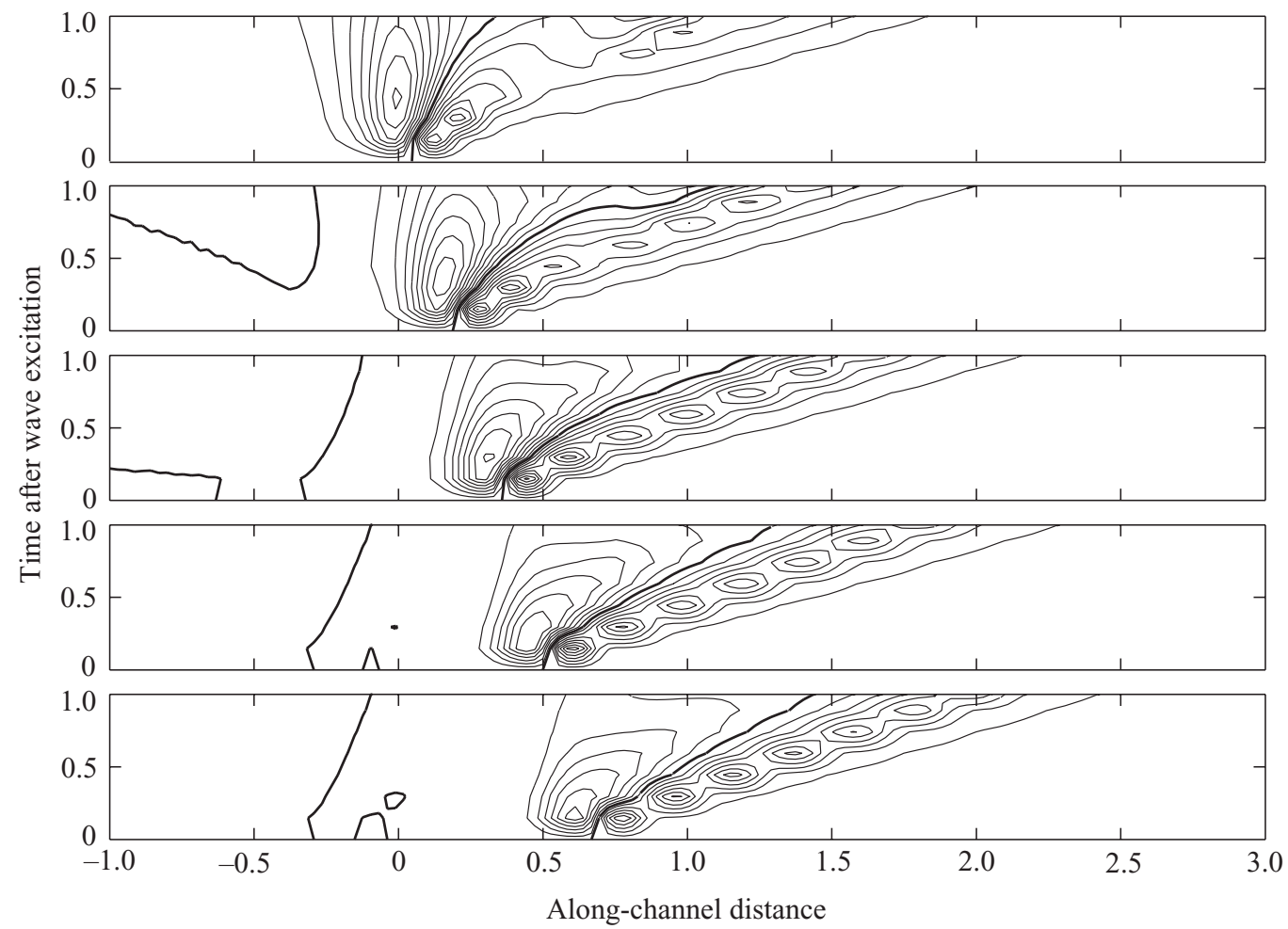

FigurE 9. The temporal development of the horizontal velocity field resulting from wave excitation experiments, waves being excited in a narrow vicinity around $x=0,0.14,0.28,0.42$ and 0.56 (upper to lower panel). The contours are of constant $u$ at intervals of $2.5 \times 10^{-3}$, the thick line being zero, and velocities below and above zero are to the left and to the right of the thick line, respectively.

flow shown in figure 7. Beginning at an upstream section $x=x_{0}$, equations (2.1)-(2.3) are integrated downstream using $d\left(x_{0}\right), u\left(x_{0}\right)$ and $g^{\prime}\left(x_{0}\right)$ equal to the vertically averaged values computed from the numerical model. The procedure also uses $w_{e}(x)$ and $u_{1}$ as computed in the numerical simulation, although the latter is so small as to have negligible effect on the outcome. As shown in the depictions of interface elevation (figure 10), the solution from the slab model (upper curve) remains subcritical, whereas the flow in the continuous model (middle curve) spills over the obstacle and becomes hydraulically supercritical. An arrow indicates the approximate point of criticality for the continuous flow based on the wave speed calculations of the previous section.

It is natural to ask whether the upstream conditions could be adjusted slightly in order to produce a solution to (2.1)-(2.3) that more closely resembles the continuous model. We fixed the values of $d\left(x_{0}\right)$ and $g^{\prime}\left(x_{0}\right)$, and increased the flow rate $q\left(x_{0}\right)=d\left(x_{0}\right) u\left(x_{0}\right)$ until the resulting solution underwent a critical transition over the obstacle. It was necessary to increase $q\left(x_{0}\right)$ by $51 \%$ to achieve this end and the resulting solution (lower curve with the dashed extension in figure 10) is therefore quite different from what is observed in the model.

\section{Incorporating vertically varying velocity and density into the layer formulation}

In order to identify the sources of the inconsistency between the layer and continuous models, it is helpful to reconsider the development of the layer formulation 


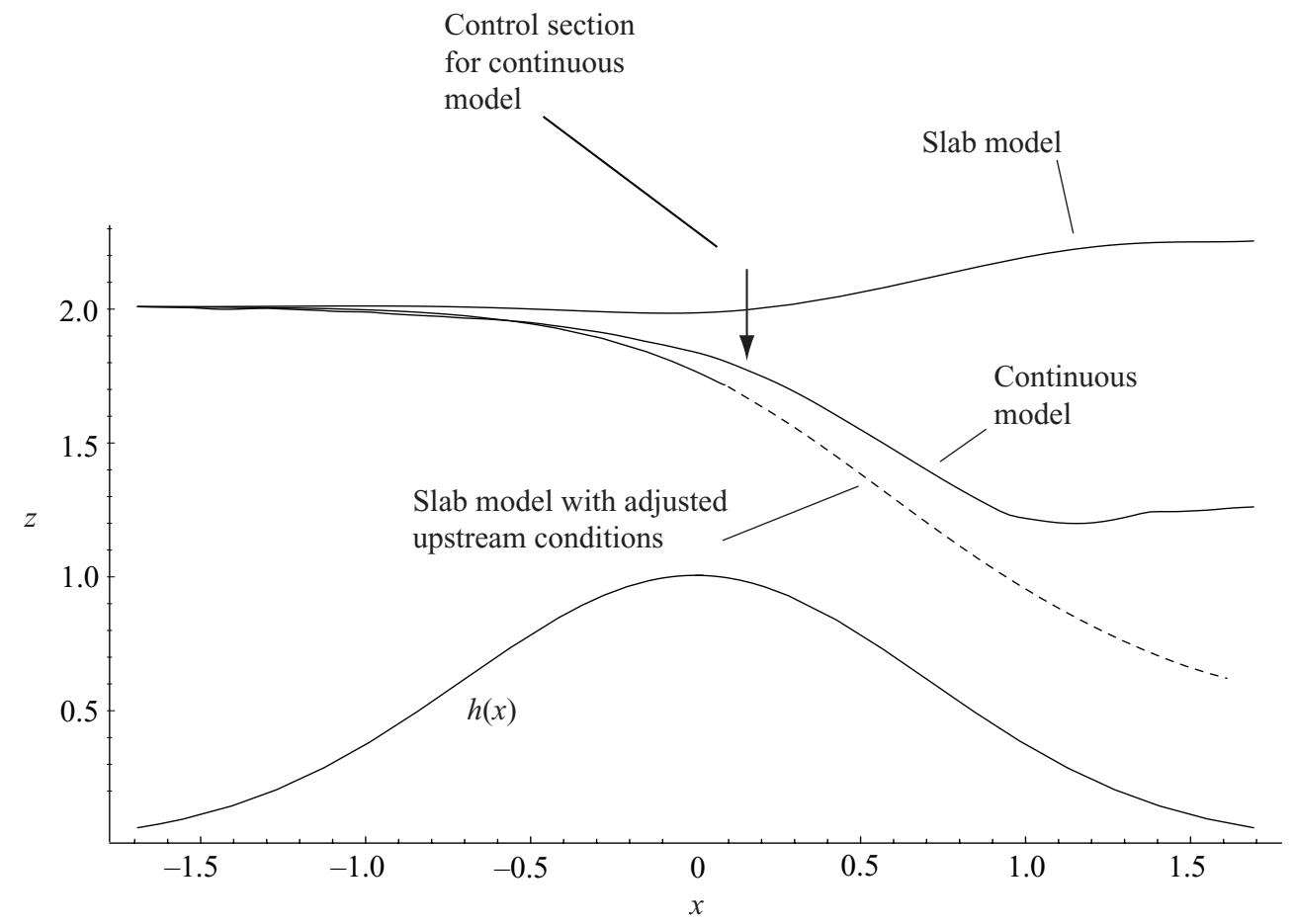

FIGURE 10. Comparison of the interface heights for the layer model and continuous model with identical upstream conditions (upper two curves). The common upstream conditions are imposed at $x_{0}=-1.69$ and here $d=1.947, u_{2}=0.185, F^{2}=0.018, u_{2} d=0.361$ and $g^{\prime}=1$. Also shown is a layer model solution in which the upstream layer depth and reduced gravity is the same as for the upper curve, but where the upstream value of $u d$ has been increased by $51 \%$. The dashed portion of this curve indicates $F>1$.

in the presence of density and velocity fields that vary continuously in the vertical. Consider a steady continuously stratified and sheared Boussinesq hydrostatic flow that takes place beneath an inactive and homogeneous upper fluid (figure 11). The upper fluid may have a uniform velocity $u_{1}$, but is otherwise inactive. We further partition the (dimensional) velocity and density in the overflowing layer into vertical averages and departures from the average:

$$
u_{2}(x, z)=\bar{u}_{2}(x)+u^{\prime}(x, z), \quad \rho_{2}(x, z)=\bar{\rho}_{2}(x)+\rho^{\prime}(x, z) .
$$

If the budgets of momentum, volume and mass are considered for a lower-layer control volume of length $\mathrm{d} x$, and $\mathrm{d} x$ is taken to zero, the following conservation laws result:

$$
\begin{gathered}
\frac{\mathrm{d}}{\mathrm{d} x}\left[d \bar{u}_{2}^{2}+\int_{h}^{h+d} u^{\prime 2} \mathrm{~d} z+\frac{1}{2} \bar{g}^{\prime} d^{2}+\frac{g}{\rho_{0}} \int_{h}^{h+d} \int_{z}^{h+d} \rho^{\prime}\left(x, z^{\prime}\right) \mathrm{d} z^{\prime} \mathrm{d} z\right]=-\bar{g}^{\prime} d \frac{\mathrm{d} h}{\mathrm{~d} x}+w_{e} u_{1} \\
\frac{\mathrm{d}}{\mathrm{d} x}\left[d \bar{u}_{2}\right]=w_{e} \\
\frac{\mathrm{d}}{\mathrm{d} x}\left[\bar{\rho}_{2} d \bar{u}_{2}+\int_{h}^{h+d} \rho^{\prime} u^{\prime} \mathrm{d} z\right]=\rho_{1} w_{e}
\end{gathered}
$$



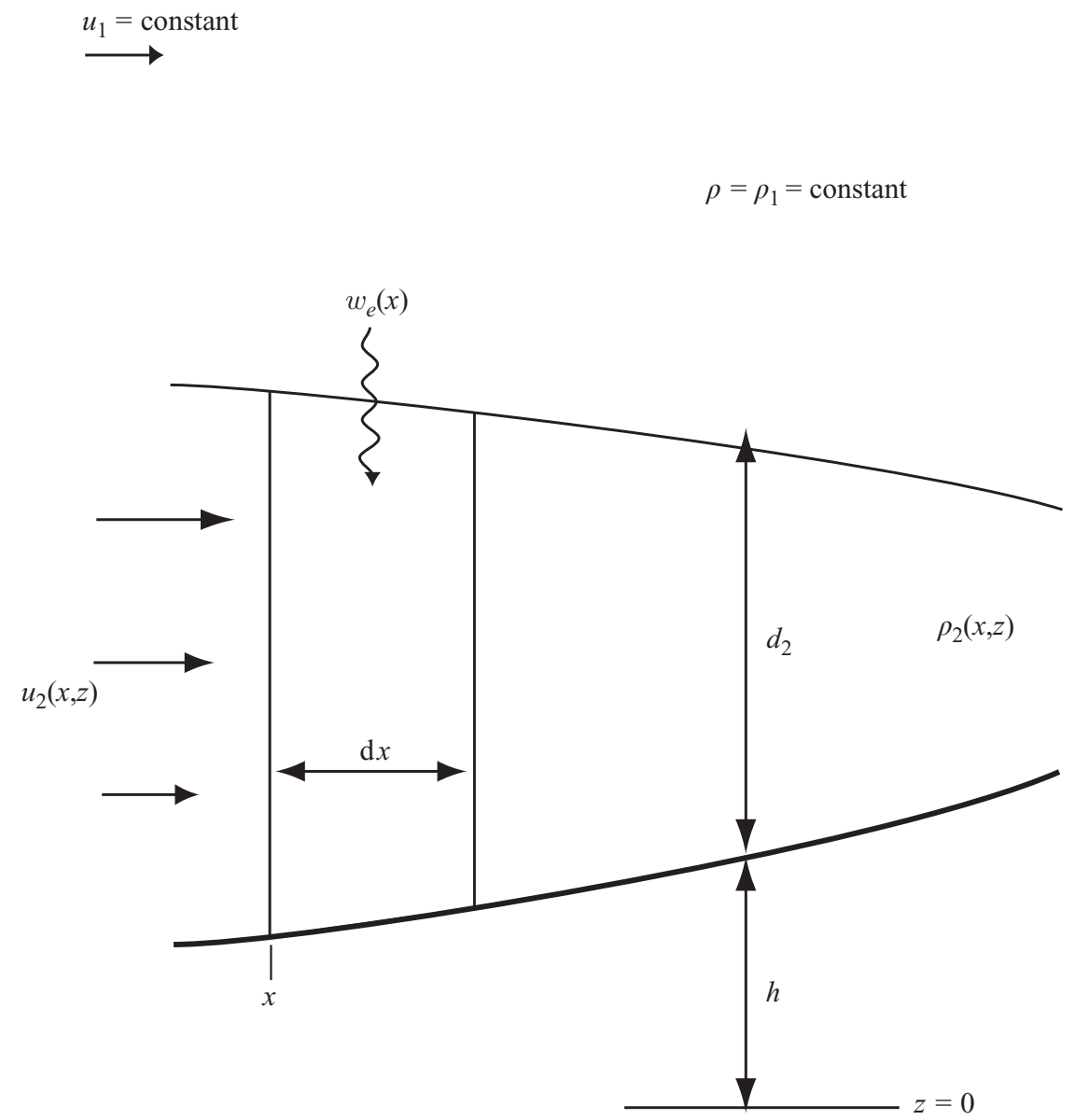

FIGURE 11. Definition sketch showing control volume extending from the bottom of the channel (heavy line) to a bounding isopycnal, across which there is a positive downwards entrainment velocity $w_{e}$. The width of the control volume is $\mathrm{d} x$. The overlying fluid is assumed to have constant density $\rho_{1}$ and velocity $u_{1}$.

where $\bar{g}^{\prime}(x)=g\left(\bar{\rho}_{2}(x)-\rho_{1}\right) / \rho_{1}$. If the product of $\rho_{1}$ and (6.2) is subtracted from the last equation, the result is a statement of conservation of buoyancy flux:

$$
\frac{\mathrm{d}}{\mathrm{d} x}\left[\left(\bar{\rho}_{2}-\rho_{1}\right) d \bar{u}_{2}+\int_{h}^{h+d} \rho^{\prime} u^{\prime} \mathrm{d} z\right]=0 .
$$

Equations (6.1)-(6.3) differ from those in the continuous model only in the neglect of effects resulting from the non-uniformity of the overlying fluid, non-hydrostatic pressure and internal friction. For each run, it has been verified that each equation is generally valid to a high degree of accuracy, implying that these effects are quite weak in our simulations.

In the absence of the primed quantities, (6.1)-(6.3) form the standard shallowwater equations for the depth-averaged velocity and density (the basis of (2.1)-(2.3)). Departures from the slab model result from vertical non-uniformities in the density and horizontal velocity. In engineering applications (e.g. Chow 1959), the departure of the momentum flux from $d \bar{u}^{2}$ in (6.1) is sometimes regarded as a correction to the 
momentum flux of the mean flow, as measured by the 'Coriolis' coefficient $\alpha$ :

$$
d \bar{u}_{2}^{2}+\int_{h}^{h+d} u^{\prime 2} \mathrm{~d} z=\alpha d \bar{u}_{2}^{2}
$$

where

$$
\alpha=\frac{d \bar{u}_{2}^{2}+\int_{h}^{h+d} u^{\prime 2} \mathrm{~d} z}{d \bar{u}_{2}^{2}}=\frac{\int_{h}^{h+d} u_{2}^{2} \mathrm{~d} z}{d \bar{u}_{2}^{2}} .
$$

Coefficients measuring the departures from the shallow-water approximations of the horizontal pressure force and the density flux can be introduced in a similar way:

$$
\frac{1}{2} \bar{g}^{\prime} d^{2}+\frac{g}{\rho_{0}} \int_{h}^{h+d} \int_{z}^{h+d} \rho^{\prime}\left(x, z^{\prime}\right) \mathrm{d} z^{\prime} \mathrm{d} z=\beta \frac{1}{2} \bar{g}^{\prime} d^{2},
$$

where

$$
\beta(x)=\frac{2 \int_{h}^{h+d} \int_{z}^{h+d}\left(\rho_{2}\left(x, z^{\prime}\right)-\rho_{1}\right) \mathrm{d} z^{\prime} \mathrm{d} z}{d^{2}\left(\bar{\rho}_{2}(x)-\rho_{1}\right)},
$$

and

$$
\left(\bar{\rho}_{2}-\rho_{1}\right) d \bar{u}_{2}+\int_{h}^{h+d} \rho^{\prime} u^{\prime} \mathrm{d} z=\gamma\left(\bar{\rho}_{2}-\rho_{1}\right) \mathrm{d} \bar{u}_{2},
$$

where

$$
\gamma(x)=\frac{\int_{h}^{h+d}\left(\rho_{2}(x, z)-\rho_{1}\right) u(x, z) \mathrm{d} z}{\left(\bar{\rho}_{2}(x)-\rho_{1}\right) d(x) \bar{u}_{2}(x)} .
$$

The coefficients $\alpha, \beta$ and $\gamma$ can be regarded as shape functions for the vertical profiles of lower-layer velocity, pressure anomaly and flux of density anomaly. (Note that our $\alpha$ is Chow's $\beta$.)

The equations for conservation of momentum and buoyancy flux anomaly can now be written as

$$
\frac{\mathrm{d}}{\mathrm{d} x}\left[\alpha d \bar{u}^{2}+\frac{1}{2} \beta \bar{g}^{\prime} d^{2}\right]=-\bar{g}^{\prime} d \frac{\mathrm{d} h}{\mathrm{~d} y}+w_{e} u_{1}
$$

and

$$
\frac{\mathrm{d}}{\mathrm{d} x}\left[\gamma \bar{g}^{\prime} d \bar{u}_{2}\right]=0
$$

and these along with (6.2) have a superficial resemblance to the flux form of the shallow-water equations. The latter are recovered when $\alpha=\beta=\gamma=1$ and departures therefore give an indication of the error incurred by treating the lower layer as a slab. From the final expression in (6.5), it is easily seen that $\alpha \geqslant 1$, and it can also be shown (Appendix B) that $\beta \leqslant 1$, provided the stratification is stable. Vertical variations of velocity and density therefore have the effect of enhancing the inertia 


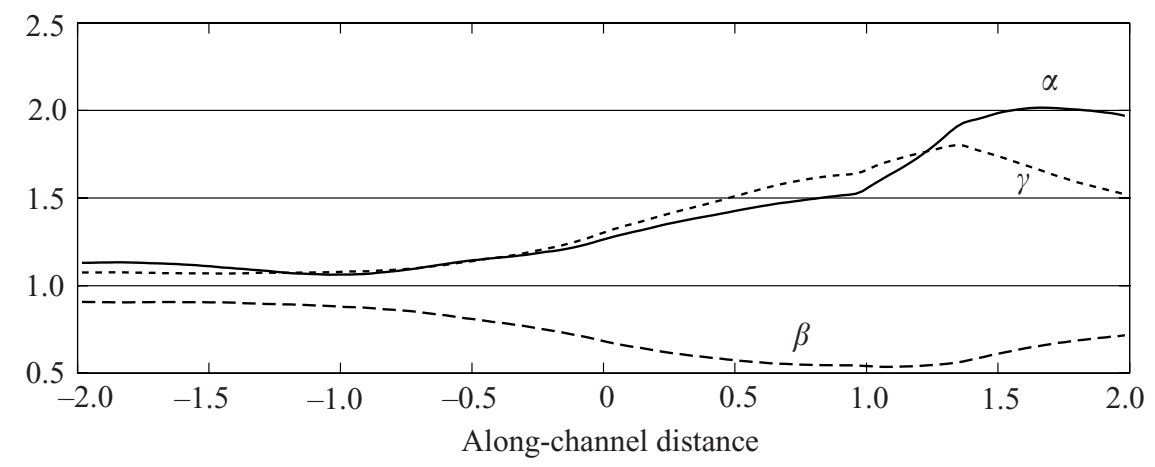

FIGURE 12. The shape functions $\alpha, \beta$ and $\gamma$ as defined by (6.5), (6.7) and (6.9), for the model output shown in figure 7.

and decreasing the buoyancy, relative to the mean flow, of the lower layer. The underestimation of the inertia term $(\alpha \geqslant 1)$ is discussed by Chow (1959) and is familiar to investigators of homogeneous open-channel flows. The new and more subtle result is the overestimation of the buoyancy term $(\beta \leqslant 1)$ by the slab model. The range of the correction $\gamma$ to the buoyancy flux is less restricted; however, it can be shown that, when the stratification is stable and the horizontal velocity decreases monotonically downward (upward) through the lower layer, $\gamma<1(>1)$. This result is also derived in Appendix B. As shown in figure 12, the values of $\alpha(x), \beta(x)$, and $\gamma(x)$ for the numerical run of figure 7 conform to these ranges and suggest significant departures from shallow-water theory. For example, the value of $\alpha(x)$ becomes as high as 1.99 in the supercritical part of the flow, whereas $\beta(x)$ becomes as low as 0.55 . The underestimation of the inertia-to-buoyancy ratio is the primary reason that the layer model solution (top curve in figure 10) is more subcritical than the full model solution.

In order to solve (6.2), (6.10) and (6.11), under general circumstances, a closure scheme would be required to relate the shape coefficients to the mean quantities. This difficulty is avoided if the density and horizontal velocity are self-similar $\left[\rho_{2}\right.$ and $u_{2}$ are functions of $(z-h(x)) / d(x)$ only], in which case $\alpha, \beta$ and $\gamma$ are constant. If this be the case, or if $\alpha, \beta$ and $\gamma$ vary gradually on the scale of the other forcing, (6.2), (6.10) and (6.11) can be used to show that

$$
\begin{aligned}
& \frac{\mathrm{d} \bar{u}_{2}}{\mathrm{~d} x}=\frac{\bar{F}^{2}}{\left(\beta-\alpha \bar{F}^{2}\right) d}\left\{w_{e}\left(\alpha+\frac{\beta}{2 \bar{F}^{2}}-\frac{u_{1}}{\bar{u}_{2}}\right)+\frac{\bar{u}_{2}}{\bar{F}^{2}} \frac{\mathrm{d} h}{\mathrm{~d} x}\right\}, \\
& \frac{\mathrm{d}(d)}{\mathrm{d} x}=\frac{\bar{F}^{2}}{\left(\beta-\alpha \bar{F}^{2}\right)}\left\{2 \frac{w_{e}}{\bar{u}_{2}}\left(\frac{\beta}{4 \bar{F}^{2}}+\frac{u_{1}}{2 \bar{u}_{2}}-\alpha\right)-\frac{1}{\bar{F}^{2}} \frac{\mathrm{d} h}{\mathrm{~d} x}\right\}
\end{aligned}
$$

and

$$
\frac{\mathrm{d} \bar{F}^{2}}{\mathrm{~d} x}=\frac{3 \bar{F}^{2}}{\left(\beta-\alpha \bar{F}^{2}\right) d}\left\{\frac{w_{e}}{\bar{u}_{2}}\left(\alpha \bar{F}^{2}+\frac{\beta}{2}-\frac{u_{1}}{\bar{u}_{2}} \bar{F}^{2}\right)+\frac{\mathrm{d} h}{\mathrm{~d} x}\right\},
$$

where $\bar{F}^{2}=\bar{u}_{2} /\left(\bar{g}^{\prime} d\right)$. Note that these relations are independent of $\gamma$ and that they reduce to (2.1)-(2.3) when $\alpha=\beta=1$. It is apparent that hydraulic criticality under the assumed conditions corresponds to

$$
\bar{F}^{2}=\frac{\beta}{\alpha} \leqslant 1
$$

Under conditions of self-similarity, critical flow occurs where the ordinary Froude number indicates subcriticality. 
In general, self-similarity is not a property of the flow field and (6.15) is invalid. For the special case of uniform density $(\beta=1)$, Garrett \& Gerdes (2003) have shown that the vertical velocity profile cannot be self-similar without a special unrealistic form for the internal dissipation. However, Wood (1968) has shown that self-similarity can occur in the presence of stratification, provided the channel bottom remains horizontal and the flow is forced entirely by sidewall contractions. Wood's solution takes the form $\rho_{2}=\rho_{2}\left(z / d_{2}(y)\right)$ and $u_{2}=u_{2}\left(z / d_{2}(y)\right)$, and examples of corresponding flows have been reproduced in the laboratory (Armi \& Williams 1993). The precise forms of these functions and the corresponding constant values of $\alpha, \beta$ and $\gamma$ depend on the upstream conditions.

In the numerical solution under discussion, which is not self-similar, $\bar{F}^{2}=1$ at $x=0.56$, whereas $\bar{F}^{2}=\beta / \alpha$ at $x=0.12$ (see figure $8 c$ ). The direct calculation of wave speed (figure 9) indicates the actual section of criticality near $x=0.14$, where $\bar{F}^{2}<1$. Positions of predicted and measured critical sections for all the numerical experiments are summarized in figure 13. There are three series of runs, each with a particular value of $h_{s} / L$, and the corresponding results are displayed in different frames. Within each series, the upper-layer velocity $u_{1}$ is varied. The position of the control section in each case, as determined by the introduction of free waves, is indicated by a horizontal line that reflects the uncertainty in visually isolating the exact location. The position at which $\bar{F}^{2}=1$ (filled circle) lies well downstream of this position in each case. We have also indicated the position at which $\bar{F}^{2}=\beta / \alpha$ (crosses), and this lies within the range of uncertainty of the direct measurement in each case.

$\bar{F}^{2}$ did not reach unity at any section in some of the experiments, and these are indicated by the absence of a filled circle in figure 13. This result has implications for certain ocean overflows that appear to be hydraulically controlled, but lack observations indicating $\bar{F}^{2} \geqslant 1$. One example is the Bab al Mandab, where measurements of the composite Froude number (the two- or three-layer extension of $\bar{F}^{2}$ ) at the main sill and narrowest section give values <1 (Pratt et al. 1999).

As $u_{1}$ decreases, the control section moves in the downstream direction, as expected from (2.6). Note that for an upper-layer velocity $u_{1}<-0.15$, the experiments with the least steep topography (lower frame) did not become critical at any point. For the steepest topography (upper frame) the influence of the upper-layer velocity is less clear; in fact, the control point appears to move slightly upstream as $u_{1}$ is decreased below -0.4 . The control is located downstream of the sill $(x=0)$ in nearly all cases. Although (2.6) predicts an upstream location for sufficiently large positive $u_{1}$, we did not check this. The required upper-layer velocity would imply a significant contribution to the composite Froude number, and so the upper layer would no longer be inactive.

Our finding that critical flow occurs near the location where $\bar{F}^{2}=\beta / \alpha$ seems consistent with the fact that vertical variations of $u$ and $\rho$ in the lower layer enhance the inertial character of the flow relative to that deduced from the vertical mean. However, the work of Garrett \& Gerdes (2003) suggests that the actual situation may be more subtle. Their study of a homogeneous free-surface shear flow, essentially the case $\beta=\gamma=1$ and $\alpha>1$, shows that the critical section indicated by direct calculation of the long-wave speed lies where $\bar{F}^{2}>1$, as opposed to the position of $\bar{F}^{2}=1 / \alpha<1$ predicted for a self-similar flow. The real critical section therefore lies downstream of the location suggested by (6.15). Garrett (2004) further argues that the presence of bottom drag pushes the critical section downstream, whereas internal friction displaces it back upstream. In the present experiments, which contain internal friction but no bottom drag, it is possible that the critical section lies upstream of where it 

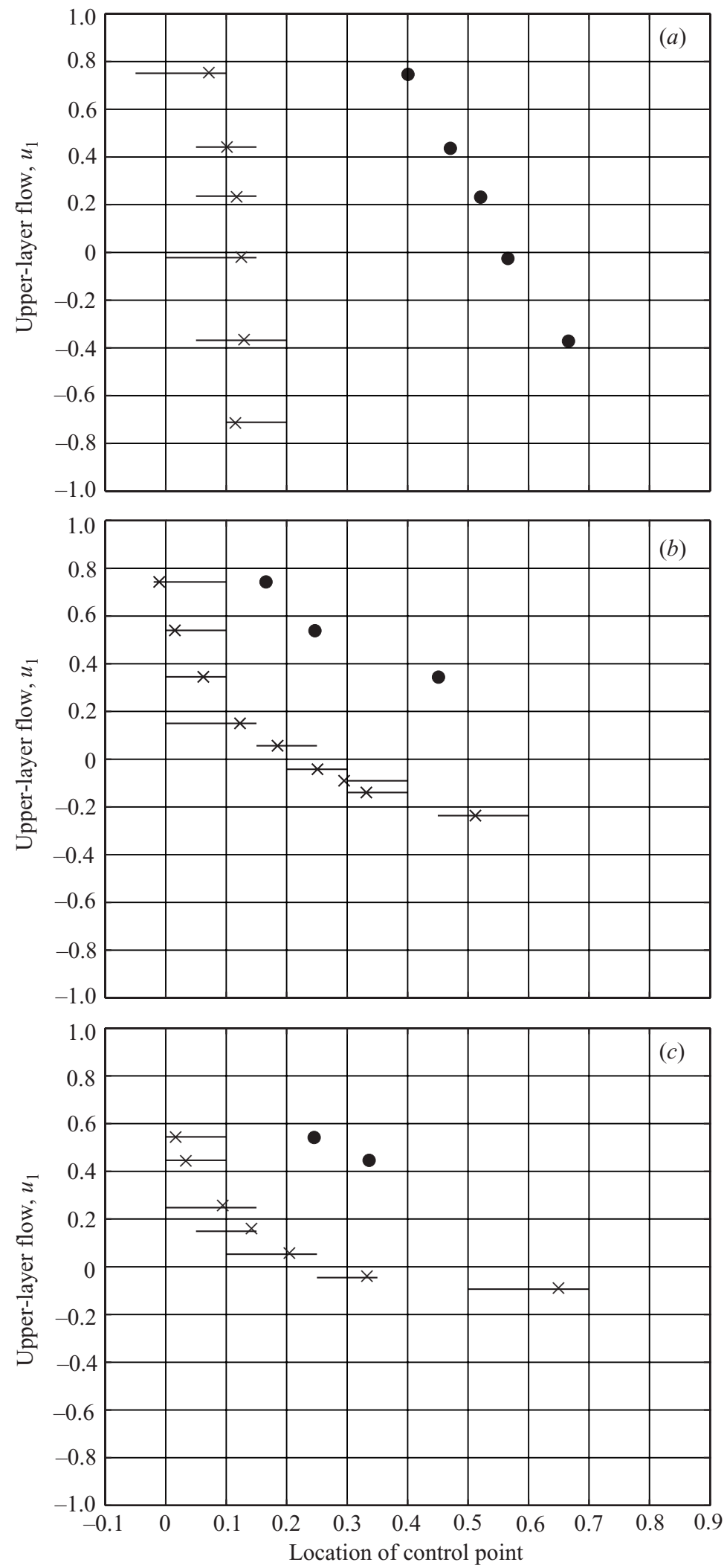

FIGURE 13. Location of the control point as a function of the upper-layer velocity. Three different aspect ratios have been used: $(a) h_{s} / L=1.41 \times 10^{-1},(b) 3.54 \times 10^{-2}$, and (c) $1.77 \times 10^{-2}$. The crosses indicate the location of $\bar{F}^{2}=\beta / \alpha$ and the filled circles show where $\bar{F}^{2}=1$. The horizontal bars show the results of the wave excitation experiments, indicating the range within which the control is located. 
would otherwise occur and that this upstream displacement brings it into proximity of the location where $\bar{F}^{2}=\beta / \alpha$.

\section{Discussion}

We have investigated the hydraulic behaviour of a dense layer of fluid flowing over an obstacle and subject to entrainment of mass and momentum from a dynamically inactive overlying fluid. If the overflow is hydrostatic and vertically homogeneous in density and horizontal velocity, the shallow-water equations can be used along with an interfacial entrainment velocity to predict the hydraulic behaviour of the flow (Gerdes et al. 2002). For example, it can be shown that hydraulic control occurs where $F^{2}=1$ and that the location of the control section may be shifted upstream or downstream from its usual position at an obstacle crest, depending on the direction and strength of the upper-layer flow. These properties have been illustrated here using an entrainment velocity based on the parameterization of Ellison \& Turner (1959). This shallow-water (slab) formulation assumes that mass and momentum are completely and instantaneously mixed downwards over the whole depth of the lower layer. We have also performed a series of numerical experiments with a non-hydrostatic model of a continuously stratified exchange flow. An overflowing lower layer has been identified and its bounding interface has been defined in order to make comparisons with the slab model. The approximate locations of critical sections have been determined by introducing small-amplitude waves into the steady states.

Some of the trends predicted by the shallow-water theory are observed in the continuous model. One is the downstream movement of the control section in response to increasing reverse velocities in the overlying fluid. On the other hand, we find poor quantitative agreement when the slab model is integrated using the upstream conditions and $w_{e}$ distribution based on a hydraulically controlled solution to the continuous model. The slab model predicts solutions substantially more subcritical than the continuous model. In fact, it was necessary to increase the upstream volume flux of the former by over $50 \%$ in order to force the shallow-water solution into a controlled state. Also, the control point in the continuous model is consistently found to lie a significant distance upstream of the point at which $F^{2}=1$.

The discrepancy between the slab and continuous models is primarily due to the strong vertical variation in density and horizontal velocity that occurs in the latter. This vertical non-homogeniety is due, in part, to the incomplete mixing of mass and momentum over the depth of the lower layer. The effects of vertically non-homogeneous $g^{\prime}$ and $u$ can be quantified by recasting the equations governing hydrostatic continuously stratified shear flow using shape functions $\alpha, \beta$ and $\gamma$ for the vertical distributions of $u, g^{\prime}$ and $g^{\prime} u$. It can be shown that $\alpha \geqslant 1$ and that (for stable stratification) $\beta \leqslant 1$, implying that inertia is underestimated and buoyancy overestimated by shallow-water theory based on the layer average $u$ and $g^{\prime}$. If the flow is self-similar, $\alpha, \beta$ and $\gamma$ are constants and it can be shown that the control point lies where $\bar{F}^{2}=\beta / \alpha<1$, which can be significantly less than unity in our simulated flow. For a range of upper-layer velocity and obstacle aspect ratio, we found good agreement between the locations of $\bar{F}^{2}=\beta / \alpha$ and where the wave excitation experiments showed that the controls were located. The agreement occurs despite the fact that $\alpha$ and $\beta$ vary as rapidly as the topography.

These findings are quite the reverse of what Garrett \& Gerdes (2003) find for homogeneous shear flow with a free surface $(\beta=1)$. For inviscid flow, they show that 
(a)

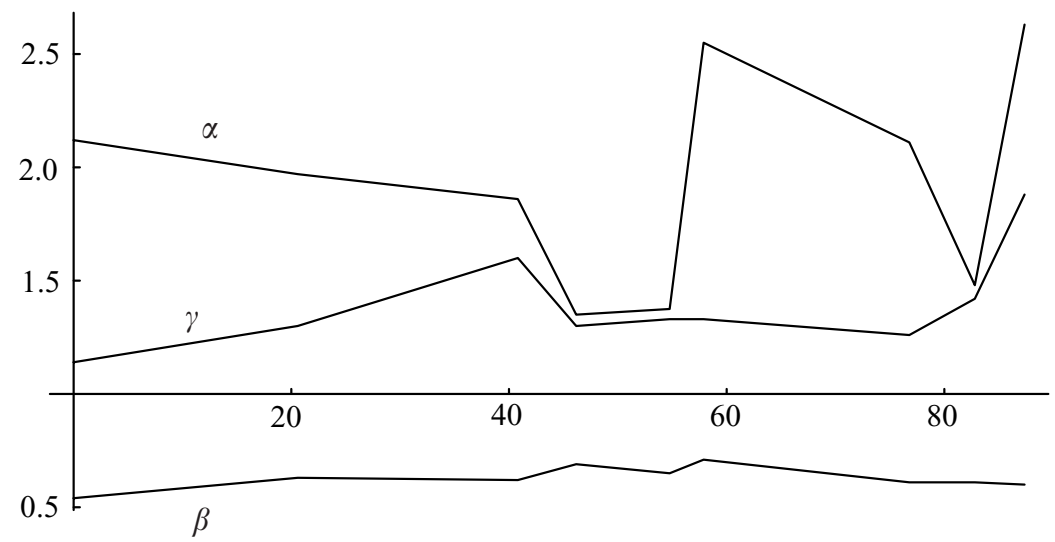

(b)

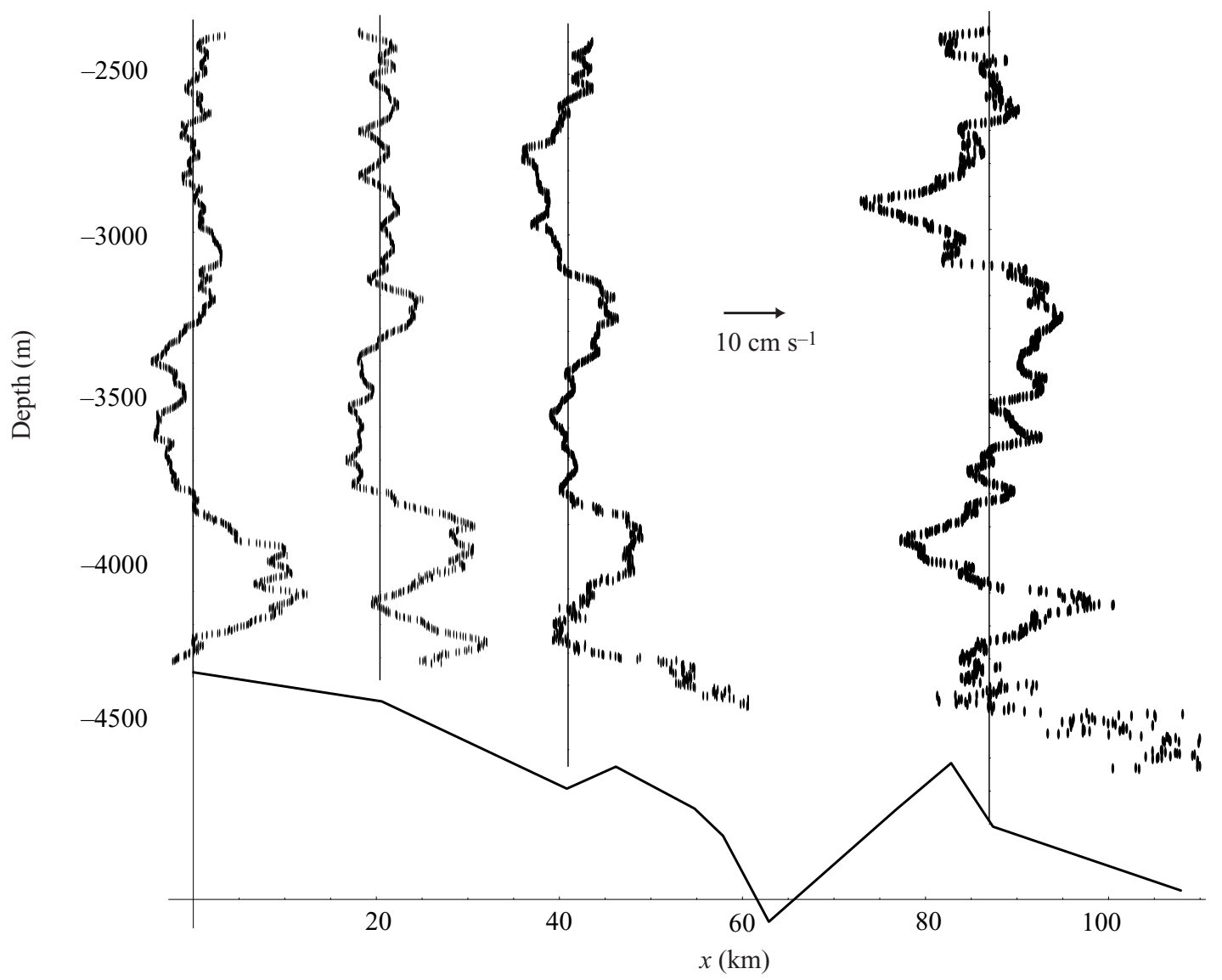

Figure 14. (a) The shape coefficients $\alpha, \beta$ and $\gamma$, calculated at nine locations along the axis of the Romanche fracture zone. $(b)$ Four of the velocity profiles used in the calculation. The outflowing layer is generally defined to extend from the bottom up to the first zero crossing of the velocity. Data provided by K. Polzin.

the control section lies where $\bar{F}^{2} \geqslant 1$, whereas the prediction based on the assumption of self-similarity would yield $\bar{F}^{2}=1 / \alpha \leqslant 1$. The apparent paradox can be removed by demonstrating that self-similarity cannot occur without the addition of an unrealistic viscous term. When the latter is included in the long-wave speed calculation, the control condition $\bar{F}^{2}=1 / \alpha$ is recovered. Garrett (2004) further shows that internal 
friction parameterized by a vertical eddy viscosity tends to move the critical point upstream from where it would otherwise be. Our case differs somewhat in that an inviscid similarity solution does exist (though only in the presence of a pure width contraction) and thus there are examples where $\bar{F}^{2}=\beta / \alpha$ gives the correct critical condition without the aid of internal dissipation. In our numerical solutions, which are not self-similar, $\bar{F}^{2}=\beta / \alpha$ also appears to give a good approximation of the observed critical section. This agreement could be because variations in $\alpha, \beta$, and $\gamma$ are unimportant or could be a fortuitous consequence of internal dissipation, acting in the way described by Garrett (2004).

For the extended model (6.2), (6.10) and (6.11) to be used in a prognostic way, $\alpha, \beta$ and $\gamma$ would have to be parameterized in terms of the average flow properties. It is probably just as easy to run a model with full stratification and mixing. The primary value of the model is diagnostic: the departures of $\alpha, \beta$ and $\gamma$ from unity provide a measure of departure from a slab model. An example from the Bab al Mandab can be calculated by treating the outflow as a single layer. Using the direct measurements of velocity and density shown in figures 6 and 10 of Pratt et al. (1999), we find $\alpha=1.12, \beta=0.77$ and $\gamma=1.11$ at the sill and $\alpha=1.11, \beta=0.64$ and $\gamma=1.15$ at the narrowest section. Data downstream of either section, where the flow may become supercritical, is unavailable. A more complete example can be calculated using data from the Romanche fracture zone (Polzin et al. 1996; Ferron et al. 1998), a deep strait that lies close to the equator and is aligned more or less east-west. The shape coefficients (figure 14a) are computed at nine stations beginning at the main sill ( $x=0$ in figure $14 b$ ) and proceeding eastward (to the right). The velocity profiles at four of the stations are shown and these reveal the overflow as a layer of intensified eastward (left-to-right) velocity extending 500-800 $\mathrm{m}$ off the bottom. Some of the velocity profiles are quite wiggly and this contributes to values of $\alpha$ and $\gamma$ that differ considerably from unity and that vary rapidly as the flow descends over the irregular bottom. The stratification (not shown) is smoother and contributes to a relatively consistent value $0.55<\beta<0.7$. The departures of the shape coefficients from unity are not significantly different from what is observed in the numerical model (figure 12) over the range $0.5<x<2$, with $\alpha$ as large as $2.0, \beta$ as small as 0.55 and $\gamma$ as large as 1.75 in both cases. A slab model would clearly not be suitable for this application.

This work is far from being the final word on the problem of ascertaining the position of a critical section of stratified shear flow. The most efficient and consistent method for doing so is still unclear, whether it be with a numerical model or field data.

M.H.N. would like to thank the Danish Natural Science Research Council for financial support. L. P. and K.H. were supported by the Office of Naval Research under grant N00014-1-01-0167 and by the National Science Foundation under grant OCE-0132903. The authors would like to thank Chris Garrett, Sonya Legg and the anonymous reviewers for a number of helpful comments. This is Woods Hole Oceanographic Contribution 11117.

\section{Appendix A}

Characteristic form of shallow-water equations with entrainment and bottom drag. 
The equations for momentum, volume and mass conservation, allowing for entrainment and quadratic bottom drag, are given by

$$
\begin{gathered}
\frac{\partial u_{2}}{\partial t}+u_{2} \frac{\partial u_{2}}{\partial x}+g^{\prime} \frac{\partial d}{\partial x}=-g^{\prime} \frac{\partial h}{\partial x}-C_{d} \frac{u_{2}\left|u_{2}\right|}{d}+w_{e} \frac{u_{1}-u_{2}}{d}+g^{\prime} \frac{w_{e}}{2 u_{2}}, \\
\frac{\partial d}{\partial t}+\frac{\partial\left(u_{2} d\right)}{\partial x}=w_{e}
\end{gathered}
$$

and

$$
\frac{\partial \rho_{2}}{\partial t}+\frac{\partial\left(u_{2} \rho_{2}\right)}{\partial x}=w_{e} \rho_{1}
$$

where $C_{d}$ is a dimensionless drag coefficient and $g^{\prime}(x, t)=g\left(\rho_{2}(x, t)-\rho_{1}\right) / \rho_{1}$. Subtracting $\rho_{1} \times(\mathrm{A} 2)$ from (A 3) leads to (2.5), the first characteristic equation for the third-order system. The remaining two equations are obtained by taking (A 1$) \pm$ $\left(g^{\prime} / d\right)^{1 / 2} \times($ A 2$)$ and these lead, after some manipulation, to (2.4).

\section{Appendix B. Restrictions on the ranges of $\beta$ and $\gamma$}

From the definition (6.7),

$$
\beta(x)=1+\frac{2 \int_{h}^{h+d} \int_{z}^{h+d} \rho^{\prime} \mathrm{d} z^{\prime} \mathrm{d} z}{d^{2}\left(\bar{\rho}_{2}(x)-\rho_{1}\right)},
$$

so the value of $\beta$ relative to unity depends on the sign of the double integral. If the stratification is stable, $\rho^{\prime}$ must decrease monotonically from a positive value at $z=h$ to a negative value at $z=h+d$. Since $\rho^{\prime}$ has no mean over this range it follows that

$$
\int_{z}^{h+d} \rho^{\prime} \mathrm{d} z^{\prime}= \begin{cases}0 & (z=h) \\ <0 & (h<z<h+d)\end{cases}
$$

and therefore

$$
\int_{h}^{h+d} \int_{z}^{h+d} \rho^{\prime} \mathrm{d} z^{\prime} \mathrm{d} z<0 .
$$

By similar reasoning, the value of $\gamma$ relative to unity depends on the sign of

$$
\int_{h}^{h+d} \rho^{\prime} u^{\prime}\left(x, z^{\prime}\right) \mathrm{d} z
$$

where $\rho^{\prime}$ again decreases monotonically, positive and then negative values as $z$ increases from $h$ to $d+h$. Let

$$
r(z, h)=\int_{h}^{z} \rho^{\prime}\left(x, z^{\prime}\right) \mathrm{d} z^{\prime}>0 \quad(h<z<h+d),
$$

and note that $r(h+d, h)=r(h, h)=0$. Then integration by parts of (B 1) leads to

$$
\int_{h}^{h+d} \rho^{\prime} u^{\prime}\left(x, z^{\prime}\right) \mathrm{d} z=-\int_{h}^{h+d} r(x, z) \frac{\partial u^{\prime}}{\partial z} \mathrm{~d} z
$$


If the total velocity $u$ increases monotonically with increasing $z$ over the lower layer, then $\partial u^{\prime} / \partial z>0$, the integral defined by (A 1) is negative, and $\gamma<1$. If $u$ decreases monotonically then $\gamma<1$.

\section{REFERENCES}

Armi, L. \& Williams, R. 1993 The hydraulics of a stratified fluid flowing through a contraction. J. Fluid Mech. 251, 355-375.

Baines, P. G. 1995 Topographic Effects in Stratified Flows. Cambridge University Press. 482 pp.

Bell, J. B., Colella, P. \& Glaz, J. M. 1989a A second-order projection method for the incompressible Navier-Stokes equations. J. Comput. Phys. 85, 257-283.

Bell, J. B. \& Marcus, D. L. 1992 A second-order projection method for variable-density flows. J. Comput. Phys. 101, 334-348.

Bell, J. B., Solomon, J. M. \& SzymczaK, W. G. $1989 b$ A second-order projection method for the incompressible Navier-Stokes equations on quadrilateral grids. AIAA 9th Comput. Fluid Dyn. Conf. Buffalo, NY, 14-16 June 1989.

Bray, N. A., OchOA, J. \& Kinder, T. H. 1995 The role of the interface layer in exchange through the Strait of Gibraltar. J. Geophys. Res. 100, 10755-10776.

Chow, V. T. 1959 Open Channel Hydraulics. McGraw-Hill.

Ellison, T. H. \& TuRner, J. S. 1959 Turbulent entrainment in stratified flows. J. Fluid Mech. 6, 423-448.

FARMer, D. M. \& Armi, L. 1986 Maximal two-layer exchange over a sill and through the combination of a sill and contraction with barotropic flow. J. Fluid Mech. 164, 53-76.

Ferron, B., Mercier, H., Speer, K., Gargett, A. \& Polzin, K. 1998 Mixing in the Romanche fracture zone. J. Phys. Oceanogr. 28, 1929-1945.

Garrett, C. \& Gerdes, F. 2003 Hydraulic control of homogeneous shear flows. J. Fluid Mech. 475, $163-172$.

Garrett, C. G. 2004 Frictional processes in straits. Deep-Sea Res. II. 51, 393-410.

Gerdes, F., Garrett, C. \& Farmer, D. 2002 On internal hydraulics with entrainment. J. Phys. Oceanogr. 32, 1106-1111.

Girton, J. B., SAnford, T. B. \& Käse, R. H. 2001 Synoptic sections of the Denmark Strait overflow. Geophys. Res. Lett. 28, 1619-1622.

Hansen, B., Turrell, W. R. \& Østerhus, S. 2001 Decreasing overflow from the Nordic seas into the Atlantic Ocean through the Faroe Bank channel since 1950. Nature 411, 927-930.

HogG, A. McC., IveY, G. N. \& Winters, K. B. $2001 a$ Hydraulics and mixing in controlled exchange flows. J. Geophys. Res. 106, 959-972.

HogG, A. McC., Winters, K. B. \& IveY, G. N. $2001 b$ Linear internal waves and the control of stratified exchange flows. J. Fluid Mech. 447, 357-375.

Ives, D. C. \& Zacharias, R. M. 1987 Conformal mapping and orthogonal grid generation. AIAA/SAE/ASME/ASEE 23rd Joint Propulsion Conf. San Diego, CA, 2 June - 29 July 1987.

Käse, R. H. \& Oschlies, A. 2000 Flow through Denmark Strait. J. Geophys. Res. 105, 28 527-28 546.

LoNG, R. R. 1954 Some aspects of the flow of stratified fluids. II Experiments with a two-fluid system. Tellus 6, 97-115.

MurraY, S. P. \& Johns, W. 1997 Direct observations of seasonal exchange through the Babal Mandab strait. Geophys. Res. Lett. 24, 2557-2560.

Pedlosky, J. 1996 Ocean Circulation Theory. Springer, 453 pp.

Polzin, K. L., Speer, K. G., Toole, J. M. \& Smitt, R. W. 1996 Intense mixing of Antarctic Bottom Water in the equatorial Atlantic Ocean. Nature 380, 54-57.

Pratt, L. J. 1986 Hydraulic control of sill flow with bottom friction. J. Phys. Oceanogr. 16, 19701980.

Pratt, L. J., Johns, W., Murray, S. P. \& Katsumata, K. 1999 Hydraulic interpretation of direct velocity measurements in the Bab al Mandab. J. Phys. Oceanogr. 29, 2769-2784.

Pratt, L. J., Deese, H. E., Murray, S. P. \& Johns, W. 2000 Continuous dynamical modes in straits having arbitrary cross sections, with applications to the Bab al Mandab. J. Phys. Oceanogr. 30, 2515-2534. 
SMAGORINSKY, J. 1963 General circulation experiments with the primitive equations. Mon. Weather Rev. 91, 99-164.

Stommel, H. M. \& Farmer, H. G. 1953 Control of salinity in an estuary by a transition. J. Mar. Res. 13, 13-20.

Winters, K. B. \& Seim, H. E. 2000 The role of dissipation and mixing in exchange flow through a contracting channel. J. Fluid Mech. 407, 265-290.

Wood, I. R. 1968 Selective withdrawal from a stably stratified fluid. J. Fluid Mech. 32, 209-223. 